\title{
DYNAMICAL FRICTION AROUND SUPERMASSIVE BLACK HOLES
}

\author{
Fabio Antonini and David Merritt \\ Department of Physics and Center for Computational Relativity and Gravitation, Rochester Institute of Technology, 85 Lomb Memorial Drive, \\ Rochester, NY 14623, USA; antonini@astro.rit.edu, merritt@astro.rit.edu \\ Received 2011 August 4; accepted 2011 November 17; published 2011 December 29
}

\begin{abstract}
The density of stars in galactic bulges is often observed to be flat or slowly rising inside the influence radius of the supermassive black hole (SMBH). Attributing the dynamical-friction force to stars moving more slowly than the test body, as is commonly done, is likely to be a poor approximation in such a core since there are no stars moving more slowly than the local circular velocity. We have tested this prediction using large-scale $N$-body experiments. The rate of orbital decay never drops precisely to zero, because stars moving faster than the test body also contribute to the frictional force. When the contribution from the fast-moving stars is included in the expression for the dynamical-friction force, and the changes induced by the massive body on the stellar distribution are taken into account, Chandrasekhar's theory is found to reproduce the rate of orbital decay remarkably well. However, this rate is still substantially smaller than the rate predicted by Chandrasekhar's formula in its most widely used forms, implying longer timescale for inspiral. Motivated by recent observations that suggest a parsec-scale core around the Galactic center (GC) SMBH, we investigate the evolution of a population of stellar-mass black holes (BHs) as they spiral into the center of the Galaxy. After $\sim 10 \mathrm{Gyr}$, we find that the density of BHs can remain substantially less than the density in stars at all radii; we conclude that it would be unjustified to assume that the spatial distribution of BHs at the GC is well described by steady-state models. One consequence is that rates of capture of BHs by the SMBH at the Galactic center (extreme-mass-ratio inspirals) may be much lower than in standard models. When capture occurs, inspiraling BHs often reach the gravitational-radiation-dominated regime while on orbits that are still highly eccentric; even after the semimajor axis has decreased to values small enough for detection by space-based interferometers, eccentricities can be large enough that the efficient analysis of gravitational wave signals would require the use of eccentric templates. We finally study the orbital decay of satellite galaxies into the central region of giant ellipticals and discuss the formation of multi nuclei and multiplet of black holes in such systems.
\end{abstract}

Key words: black hole physics - galaxies: kinematics and dynamics - Galaxy: center - gravitational waves

Online-only material: color figures

\section{INTRODUCTION}

Dynamical friction plays a central role in many astrophysical contexts. It drives the orbital inspiral and merger of satellite galaxies (e.g., Murai \& Fujimoto 1980; Ibata \& Lewis 1998; van den Bosch et al. 1999) and the formation of massive black hole binaries (e.g., Quinlan 1996; Milosavljević \& Merritt 2001; Makino \& Funato 2004), and it is the fundamental mechanism leading to mass segregation in dense stellar systems (e.g., Bahcall \& Wolf 1977; Freitag et al. 2006; Hopman \& Alexander 2006).

Chandrasekhar formulated the principle of dynamical friction under the assumptions of an infinite, homogeneous, and isotropic field of stars (Chandrasekhar 1943). Despite these simplifications, his theory has been shown to work remarkably well in a wide variety of more general situations. Dynamical friction can be understood as the drag induced on a test particle by the overdensity (i.e., the gravitational wake) that is raised behind it by the deflection of stars (Danby \& Camm 1957; Kalnajs 1972; Mulder 1983). The surprisingly good agreement between theory and numerical results may be attributed to the fact that the wake is a local structure, and over small spatial scales, the stellar background appears nearly homogeneous (Weinberg 1986). On the other hand, numerical studies have revealed a few, astrophysically important contexts in which Chandrasekhar's theory appears to break down. These include the deceleration of a rotating stellar bar (Weinberg 1985), inspiral in harmonic (constant- density) cores (Hernandez \& Gilmore 1998; Goerdt et al. 2006; Read et al. 2006; Inoue 2009), and the orbital evolution of a displaced supermassive black hole (SMBH; Gualandris \& Merritt 2008).

In this paper, we present a comprehensive study of dynamical friction in the nuclei of galaxies containing a dominant central point mass. In particular, we investigate the case of shallow density profiles around SMBHs. Such nuclei appear to be common and perhaps even generic. For instance, the luminosity profiles of bright elliptical galaxies always exhibit flat central cores (Ferrarese et al. 1994; Lauer et al. 1992). Even the Milky Way, which was long believed to have a steeply rising mass density near Sgr A*, is now believed to have a parsec-scale core (Buchholz et al. 2009; Do et al. 2009; Bartko et al. 2010). Similar models may also be applicable to dark matter halos, if the central point mass is identified with the stellar spheroid (Borriello \& Salucci 2001; Binney \& Evans 2001; Spekkens et al. 2005).

Theoretical treatments of dynamical friction make a surprising prediction about the frictional force in such systems. Essentially all of the decelerating force is predicted to come from stars that are moving more slowly than the test body. But the phase-space density of a galaxy with a shallow density cusp around a SMBH falls to zero at low energies: below a certain radius (roughly $1 / 2$ the core radius), there are no stars locally that move more slowly than the circular velocity at that radius. Chandrasekhar's formula, in its most widely used form, would 
predict no frictional force. In the case of an eccentric orbit that passes in and out of the core, the frictional force would be small near periapsis, leading to a rapid increase in orbital eccentricity-the opposite of the usual assumption. Our numerical experiments reveal that the frictional force does not drop precisely to zero in such nuclei. We show that the evolution can be well described by a more general form formula that includes a contribution to the force from stars moving faster than the test mass. In this sense, our results affirm the correctness of Chandrasekhar's physical picture, but only if the proper fieldstar velocity distribution is used (as opposed to, say, a Maxwellian), and only if the usual simplifying assumptions that lead to a neglect of the contribution of the fast stars to the frictional force are relaxed.

In Section 2, we review Chandrasekhar's derivation of the dynamical-friction force and highlight the approximations that lead to the neglect of the contribution from the fast-moving stars. We also briefly discuss alternative treatments of dynamical friction. In Section 3, we use Chandrasekhar's formulae to integrate the equations of motion of a massive body and follow its inspiral into the center of a model designed to represent the Galactic center (GC). In Section 4, we use large-scale $\mathrm{N}$-body simulations to test the theory in the case of inspiral of massive objects in a nuclear star cluster with a flat density profile. Section 5 investigates the formation of the gravitational wake in the self-consistent simulations. Applications of our results to a variety of astrophysical problems are discussed in Section 6 and Section 7 sums up.

\section{DYNAMICAL FRICTION}

The motivation for the $N$-body experiments described in this paper is the existence of physically interesting models of galactic nuclei in which the standard dynamical-friction formula predicts little, or zero, frictional force. We begin in this section by re-deriving the standard formula, noting the simplifying approximations that are usually made. We then present the more general form of Chandrasekhar's formula that includes contributions from field stars of all velocities, not just those that move more slowly than the test body at infinity, and we evaluate the expected contribution from the fast-moving stars in our models. We also compute how the fast- and slow-moving stars contribute differently to the steady-state density wake, using a technique first applied by Mulder (1983). Finally, we comment on perturbative approaches to computing dynamical friction that relax the assumption of an infinite homogeneous medium. The results obtained in this section constitute a set of baselines against which the $N$-body results can be compared.

\subsection{Chandrasekhar's Treatment}

Chandrasekhar (1943) derived the coefficient of dynamical friction by summing the encounters of a test body with passing stars, assuming that the unperturbed motion of the test body was linear and unaccelerated, and that the field-star distribution was infinite and homogeneous spatially and isotropic in velocity space.

The velocity change of a test body of mass $M$ in one encounter with a field star of mass $m \ll M$ is

$$
\Delta v_{\|}=-2 V \frac{m}{M} \frac{1}{1+p^{2} / p_{0}^{2}},
$$

where $V$ is the relative velocity at infinity, $p$ is the impact parameter, and $p_{0} \equiv G M / V^{2}$. The velocity change in Equation (1) is parallel to the initial, relative velocity $\boldsymbol{V}$ before the encounter. In order to derive the coefficient of dynamical friction, one sums the velocity changes in the direction of motion of the test mass, per unit interval of time, over all impact parameters, and over all values for the relative velocity at infinity. The summation over impact parameters, at fixed $V$, is achieved by multiplying Equation (1) by $2 \pi p n V d p$, with $n$ the number density of field stars, and integrating $d p$

$$
\overline{\left(\Delta v_{\|}\right)}=-\frac{2 \pi G^{2} M m n}{V^{2}} \ln \left(1+p_{\max }^{2} / p_{0}^{2}\right) .
$$

Under the assumption that $\Lambda \equiv p_{\max } / p_{0} \gg 1$, this can be written as

$$
\overline{\left(\Delta v_{\|}\right)}=-\frac{4 \pi G^{2} M m n}{V^{2}}\left[\ln \Lambda+\frac{1}{2} \frac{p_{0}^{2}}{p_{\max }^{2}}+\cdots\right] .
$$

Terms beyond the first in brackets, the so-called non-dominant terms, are usually neglected.

Returning to the more general form (2), the dynamicalfriction coefficient is obtained by a second integration over field-star velocities $\boldsymbol{v}_{\star}$. The relative velocity is $\boldsymbol{V}=\boldsymbol{v}-\boldsymbol{v}_{\star}$, with $\boldsymbol{v}$ being the velocity of the test star. Since Equation (2) gives the velocity change in the direction of the initial relative motion, it must be multiplied by

$$
\frac{\boldsymbol{V} \cdot \boldsymbol{v}}{V v}=\frac{v-v_{\star}}{V}
$$

to convert it into a velocity change in the direction of the test star's motion, assumed here to be along the $x$-axis. Let $f\left(\boldsymbol{v}_{\star}\right) d \boldsymbol{v}_{\star}$ be the number density of field stars in velocity increment $\boldsymbol{v}_{\star}, \boldsymbol{v}_{\star}+d \boldsymbol{v}_{\star}$, normalized to unit total number. The dynamicalfriction coefficient is

$$
\begin{aligned}
\left\langle\Delta v_{\|}\right\rangle= & \int f\left(\boldsymbol{v}_{\star}\right) \overline{\left(\Delta v_{\|}\right)} \frac{v-v_{\star x}}{V} d \boldsymbol{v}_{\star}=-2 \pi G^{2} M \rho \\
& \times \int f\left(\boldsymbol{v}_{\star}\right) \frac{v-v_{\star, x}}{V^{3}} \ln \left(1+\frac{p_{\max }^{2} V^{4}}{G^{2} M^{2}}\right) d \boldsymbol{v}_{\star},
\end{aligned}
$$

where $\rho=m n$.

Henceforth, we assume that the field-star distribution is isotropic in velocity space. Following Chandrasekhar (1943), we represent the velocity-space volume element in terms of $v_{\star}$ and $V$ using

$$
v-v_{\star x}=\frac{V^{2}+v^{2}-v_{\star}^{2}}{2 v} .
$$

The result is

$$
\begin{aligned}
&\left\langle\Delta v_{\|}\right\rangle=-\frac{2 \pi^{2} G^{2} M \rho}{v^{2}} \int_{0}^{\infty} d v_{\star} v_{\star} f\left(v_{\star}\right) \mathcal{H}\left(v, v_{\star}, p_{\max }\right), \\
& \mathcal{H}\left(v, v_{\star}, p_{\max }\right)= \frac{1}{8 v_{\star}} \int_{\left|v-v_{\star}\right|}^{v+v_{\star}} d V\left(1+\frac{v^{2}-v_{\star}^{2}}{V^{2}}\right) \ln \\
& \times\left(1+\frac{p_{\max }^{2} V^{4}}{G^{2} M^{2}}\right) .
\end{aligned}
$$

(The quantity $J$ defined in Equation (26) of Chandrasekhar (1943) is equal to $8 v_{\star} \mathcal{H}$.) The integral that defines $\mathcal{H}$ turns out to have an analytic solution; the expression is complicated 
and we do not reproduce it here. Chandrasekhar (1943) gave several approximate forms for $\mathcal{H}$ valid for $p_{\max } / p_{0} \gg 1$, e.g., his Equation (30):

$$
\mathcal{H} \approx \begin{cases}\ln \frac{p_{\max }}{G M}\left(v^{2}-v_{\star}^{2}\right) & \text { if } v>v_{\star}, \\ \frac{1}{2} \ln \left(4 \frac{p_{\max }}{G M} v_{\star}^{2}\right)-1 & \text { if } v=v_{\star}, \\ \ln \left(\frac{v_{\star}+v}{v_{\star}-v}\right)-2 \frac{v}{v_{\star}} & \text { if } v<v_{\star} .\end{cases}
$$

In the standard approximation (e.g., Rosenbluth et al. 1957), the non-dominant terms are set to zero, and the velocity dependence of the logarithmic term in the integrand of Equation (6) is ignored. Instead, one writes

$$
\ln \left(1+\frac{p_{\max }^{2} V^{4}}{G^{2} M^{2}}\right)=2 \ln \Lambda \equiv 2 \ln \left(\frac{p_{\max }}{p_{\min }}\right)
$$

and the lower bound $p_{\text {min }}$ is set to $G M / v_{\star \text {,rms }}^{2}$. The weighting function $\mathcal{H}$ then takes on the simple form

$$
\mathcal{H}= \begin{cases}\ln \Lambda & \text { if } v>v_{\star}, \\ 0 & \text { if } v<v_{\star},\end{cases}
$$

and the coefficient of dynamical friction is

$$
\left\langle\Delta v_{\|}\right\rangle=-4 \pi G^{2} M \rho \times 4 \pi \int_{0}^{v} d v_{\star}\left(\frac{v_{\star}}{v}\right)^{2} f\left(v_{\star}\right) .
$$

Equation (10) reproduces the well-known result that only field stars with $v_{\star}<v$ contribute to the frictional force.

In this paper, we consider models for galactic nuclei in which the number of stars moving more slowly than the test body can be vanishingly small. In such models, one expects that a significant fraction of the frictional force might come from stars with $v_{\star}>v$.

The distribution of field-star velocities in our models has the following form within the core:

$$
f\left(v_{\star}\right)= \begin{cases}f_{0}\left(2 v_{c}^{2}-v_{\star}^{2}\right)^{\gamma-3 / 2} & \text { if } v_{\star}<2^{\frac{1}{2}} v_{c}, \\ 0 & \text { if } v_{\star}>2^{\frac{1}{2}} v_{c},\end{cases}
$$

where the normalizing constant

$$
f_{0}=\frac{\Gamma(\gamma+1)}{\Gamma\left(\gamma-\frac{1}{2}\right)} \frac{1}{2^{\gamma} \pi^{3 / 2} v_{c}^{2 \gamma}}
$$

corresponds to unit total number. This expression is equivalent to Equation (5); it gives the local distribution of velocities at a radius where the circular velocity is $v_{c}=\left(G M_{\bullet} / r\right)^{1 / 2}$, assuming the density of field stars follows $r^{-\gamma}$. The phase-space density is zero for $v_{\star} \geqslant v_{\mathrm{esc}}=2^{1 / 2} v_{c}$.

Of more interest here is the behavior of $f$ at small values of $v_{\star}$, and when $\gamma<3 / 2$; for such values of $\gamma$ the phase-space density diverges at $v_{\star}=2^{1 / 2} v_{c}$. As $\gamma \rightarrow 1 / 2$, the velocity distribution becomes progressively narrower, and in the limit, $f\left(v_{\star}\right)$ is a delta-function at $v_{\star}=2^{1 / 2} v_{c}$; in other words, all stars have zero energy. This may be seen as a consequence of the well-known fact that $\rho \propto r^{-0.5}$ is the shallowest power-law density profile consistent with an isotropic velocity distribution in a point-mass potential.

In the case of a test body moving in a circular orbit with $v=v_{c}$, the number of field stars with $v_{\star}<v$ will drop as $\gamma$ approaches $1 / 2$ and will equal zero in the limiting case $\gamma=1 / 2$. The standard dynamical-friction coefficient, Equation (10), predicts zero frictional force in this limit.

In this situation, it is clearly of interest to compute the contribution of the fast-moving stars to the total frictional force. We did this by evaluating $\mathcal{H}$ in its "exact" form, Equation (6c). Figure 1 shows the results. In addition to $\gamma$, the results depend on the parameter

$$
\ln \Lambda \equiv \ln \left(\frac{p_{\max } v_{c}^{2}}{G M_{\bullet}}\right),
$$

which plays the role of Coulomb logarithm. We note the following results.

1. For $\gamma \gtrsim 3 / 2$, the contribution to the frictional force from the fast-moving stars is negligible, particularly when $\ln \Lambda$ is also large.

2. For $\gamma \lesssim 3 / 2$, the fast-moving stars contribute a progressively larger fraction of the total frictional force, particularly when $\ln \Lambda$ is small.

3. When $\gamma=0.55$, near the limiting value, the total frictional force is small, and almost all of it comes from stars with $v_{\star}>v$.

4. Whereas the contribution to the force from the slow-moving stars depends strongly on $\gamma$, the contribution from the fastmoving stars is almost independent of $\gamma$.

According to Equation (7), the contribution of the fast stars must tend to zero as $\ln \Lambda$ is made sufficiently large. This is consistent with Figure 1 ; however, for $\gamma \approx 0.5$, the value of $\ln \Lambda$ required for the slow stars to dominate is far greater than any physically reasonable value.

\subsection{Mulder's Treatment}

The foregoing treatment highlighted the contribution of the fast-moving stars, $v_{\star}>v$, to the total frictional force. However, it did not provide much insight into why the two populations contribute in such a different way to the force. Of course, the $N$-body experiments described in this paper include both populations of stars. In the simulations, the field stars quickly establish a nearly steady-state distribution in a frame moving with the test mass - a "dynamical-friction wake" (Danby \& Camm 1957; Kalnajs 1972; Mulder 1983). The overdensity in the wake is responsible for the decelerating force that acts on the test body. A large fraction of the mass in the wake must be contributed by the fast stars, particularly in the case that the fast stars dominate the density at large distances. Why then do these stars contribute relatively little to the frictional force?

One way to address this question is via the technique of Mulder (1983). Mulder computed the steady-state distributions of stars around a moving test mass, making essentially the same assumptions as made by Chandrasekhar (1943). He did this by invoking Jeans's theorem in a frame moving with the test mass and showing that an isotropic $f\left(v_{\star}\right)$ at infinity could be expressed in terms of two of the integrals of motion in the Kepler problem. This then allowed him to compute the steadystate density, in the moving frame, at all locations around the test mass. The dynamical-friction force followed from a second integration of the density over space; Mulder showed that the results for the frictional force so obtained were consistent with Chandrasekhar's predictions, if $p_{\max }$ were associated with the maximum dimension of the spatial grid used to carry out the force integration. 


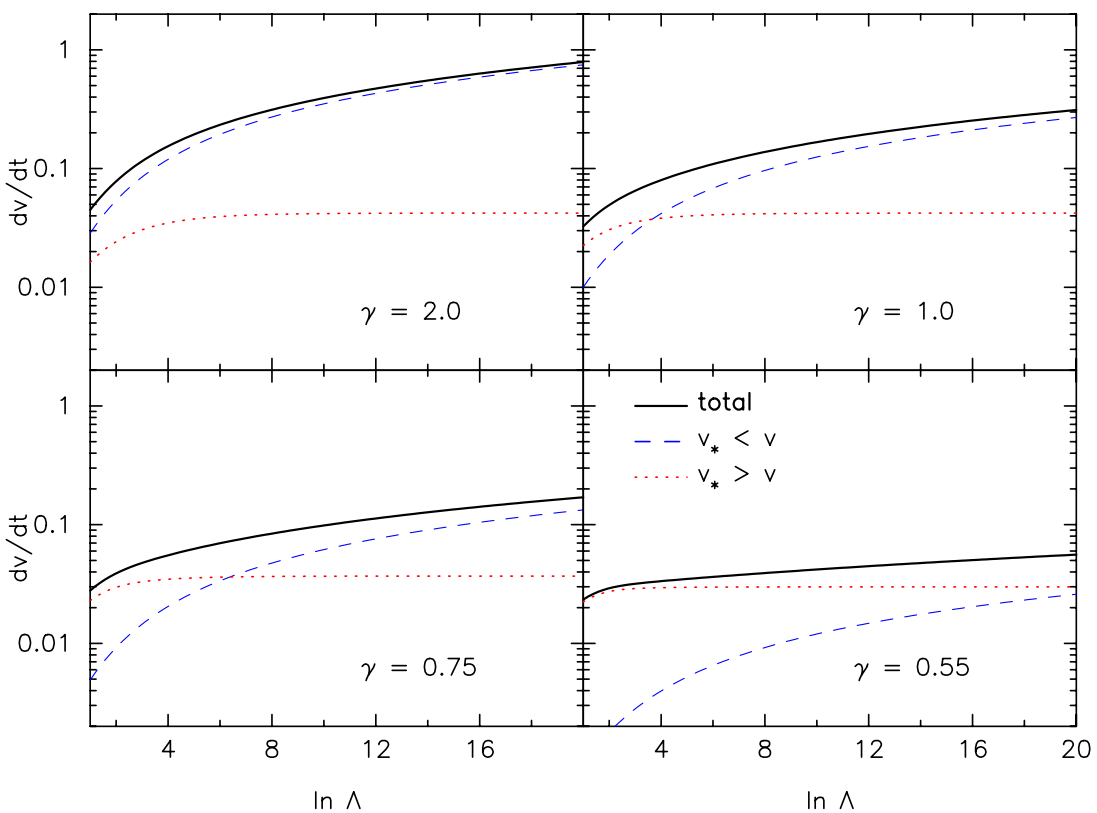

Figure 1. Contribution to the total dynamical-friction force from stars moving faster, or more slowly, at infinity than the test body, assuming the velocity distribution of Equation (11). The test body is assumed to be moving at the local circular velocity $v_{c}$. In these plots, the configuration-space density $\rho$ remains fixed as $\gamma$ is varied. (A color version of this figure is available in the online journal.)

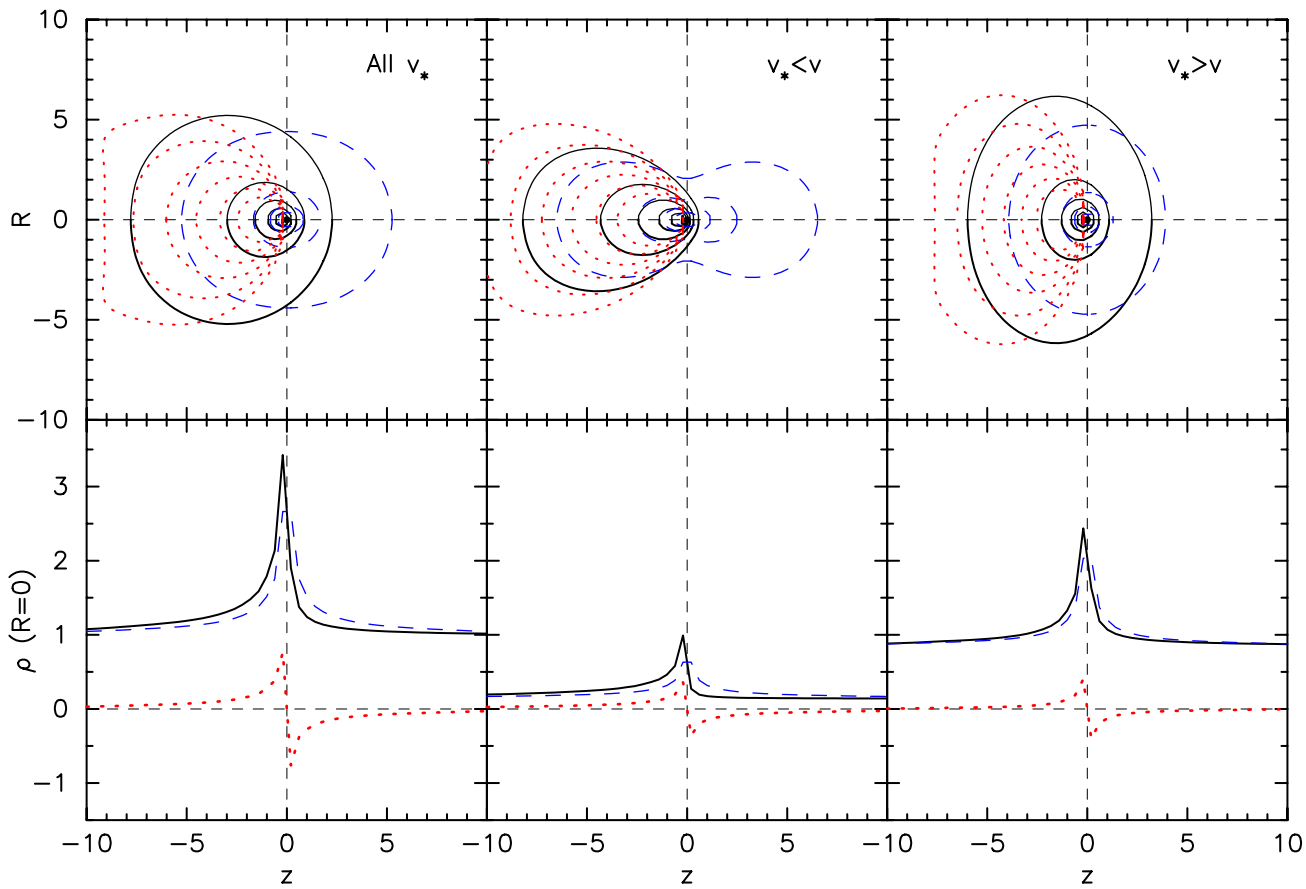

Figure 2. Dynamical-friction wakes, computed via Mulder's (1983) technique, assuming Equation (5) with $\gamma=5 / 4$ for the velocity distribution at infinity; the test mass is located at the origin and is assumed to be moving at constant velocity $v=v_{c}$, as in Figure 1. The top panels show contours of the density, in a plane that contains the test body's velocity vector; the left panel shows the total density, the middle panel shows the density contributed by the stars with $v_{\star}<v$ at infinity, and the right panel shows the contribution from stars with $v_{\star}>v$ at infinity. Black (solid) curves show the total response from the indicated stars; blue (dashed) curves show the part of the response that is symmetric with respect to $z$; red (dotted) curves show the asymmetric part (only on one side), which is responsible for the frictional force. The contours are spaced logarithmically in density and the contour spacing is different in the three panels. The lower panels show the density along the symmetry axis, i.e., along a line through the test body in the direction of its motion. Units are $G=M=v=1$.

(A color version of this figure is available in the online journal.)

Mulder's technique can be modified, to compute the separate contributions to the dynamical-friction wake of the fast $\left(v_{\star}>v\right)$ and slow $\left(v_{\star}<v\right)$ stars; here, as above, $v_{\star}$ refers to the fieldstar velocity at infinity. The results are shown in Figure 2, for $\gamma=5 / 4$. For this choice of $\gamma$, the fast stars dominate the total density at infinity. The density that they generate near the test body is also higher, everywhere along the symmetry axis, than the density due to the slow stars. However, the shapes of the two density wakes are very different: in the case of the fast stars, the wake is elongated counter to the direction of the test body's motion, while in the direction parallel to the motion, the change in density between the upstream and downstream sides of the 
test mass is much less than in the case of the wake produced by the slow stars. These two differences are responsible for the small contribution of the fast stars to the total frictional force (Figure 1), in spite of the higher density of those stars at infinity and in the wake.

Comparison of the upstream and downstream densities in Figure 2 also suggests why the relative contribution of the fast stars to the frictional force drops off with increasing $\ln \Lambda$ in Chandrasekhar's treatment (Figure 1). At large distances from the test body, the wake produced by the fast stars is nearly symmetric; the greatest asymmetry is in the region near the test mass. The wake generated by the slow stars, on the other hand, maintains its asymmetry much farther from the test body. Roughly speaking, the density far from the origin in Figure 2 is produced by stars with large impact parameters, and so increasing $p_{\max }$ in Chandrasekhar's treatment corresponds to more heavily weighting the contribution from the slow-moving stars.

\subsection{Perturbative Treatments and Inhomogeneous Systems}

In treatments like Chandrasekhar's and Mulder's, the unperturbed trajectories consist of straight lines. In reality, both test and field stars follow non-rectilinear orbits about the center of the galaxy. Chandrasekhar's theory might be expected to give approximately correct results even in this case, as long as $p_{\max } \gg p_{\text {min }}$, since over many decades in scale the orbits of the field stars will appear nearly rectilinear as seen by the test body. But given certain assumptions, perturbation theory can be used to more correctly compute the response of the orbits in a galaxy to the presence of a perturbing potential (Lynden-Bell \& Kalnajs 1972; Tremaine \& Weinberg 1984; Rauch \& Tremaine 1996). One finds that the net torque on the test mass is due to orbits near resonance, i.e., orbits for which the frequencies associated with the radial and angular motions satisfy a relation $l_{1} \omega_{r}+l_{2} \omega_{\theta}-l_{3} \Omega_{t}=0$, where the $l_{i}$ are integers and $\Omega_{t}$ is the frequency of rotation of the test mass (assumed to be on a circular orbit). The acceleration induced by the resonant orbits depends on how quickly the orbit of the test mass is evolving; if orbital decay is very slow, the influence of a single resonance can buildup, invalidating the perturbative assumption, while if it is too fast, the assumption of near-stationarity is violated. Furthermore, in a real galaxy (or $N$-body system) the frequency spectrum of the perturbing potential is not made up of sharp lines, but rather is broadened by the time dependence of the decaying orbit and by the finite age of the galaxy.

Due to the computational complexity involved, applications of this approach have so far been limited to bodies following circular orbits in simple (Plummer, scale-free) galaxy models, and the results have mostly been interpreted as corrections to the predictions of Mulder and Chandrasekhar. For instance, Weinberg (1986) emphasized the similarity in the structure of the wake as computed via the perturbation formulae and via Mulder's approach. The main element that the perturbative treatments add is a quantitative estimate of the Coulomb logarithm. Not surprisingly, none of these studies has attempted to relate the frictional force separately to the "fast" and "slow" stars as they appear in Chandrasekhar's treatment; doing so would be an ill-defined problem since all stars are included, self-consistently, in the perturbative treatments. Nevertheless, as far as we can tell, comparisons with Chandrasekhar's theory are always made via Equation (10), which ignores the fast-moving stars.
A potentially important application of the perturbative methods is to cases where the assumption of locality is violated. For instance, a satellite that orbits just outside a galaxy, where the local density is zero, would experience no frictional force if the local properties of the background were assumed to hold everywhere; in reality it feels a force due to polarization of the orbits inside the galaxy (Palmer \& Papaloizou 1985). The models considered in this paper constitute a second case where the assumption of locality may be inappropriate, since some of the frictional force acting on a test mass orbiting in the core will come from stars outside the core, where $f(\boldsymbol{v})$ has a different functional form, including (for instance) some slow-moving stars. In lieu of such a calculation (and in view of the difficulties associated with interpreting the results, e.g., Weinberg 2004), an $\mathrm{N}$-body treatment seems a logical first step. As we will see, Chandrasekhar's formula, in its more general form, turns out to reproduce the $N$-body results quite well.

\section{ORBITAL EVOLUTION BASED ON CHANDRASEKHAR'S FORMULAE}

We are interested in the orbital evolution of a massive body as it spirals in toward the center of a galaxy that contains a $\mathrm{SMBH}$. In subsequent sections, we present results from largescale, direct-summation $N$-body simulations. As a basis for comparison, we present in this section the predictions of Chandrasekhar's approximate formula. We represent the stars via a smooth, fixed potential and integrate the equations of motion of the massive body in the fixed analytic potential including a term that represents the non-conservative contribution of dynamical friction.

We base our model for the stellar density on the observed distribution of old stars at the GC. Number counts (Buchholz et al. 2009; Do et al. 2009; Bartko et al. 2010) are consistent with a density that follows a broken power law:

$$
\rho(r)=\rho_{0}\left(\frac{r}{r_{0}}\right)^{-\gamma}\left[1+\left(\frac{r}{r_{0}}\right)^{\alpha}\right]^{\left(\gamma-\gamma_{e}\right) / \alpha},
$$

where $\alpha$ is a parameter that defines the transition strength between inner and outer power laws and $r_{0}$ is the scale radius. Following Merritt (2010), we adopt $r_{0}=0.3 \mathrm{pc}, \alpha=4$, and $\gamma_{e}=1.8$ as fiducial values. The central slope $\gamma$ was left as a free parameter. The normalizing factor $\rho_{0}$ was chosen in such a way that for each value of $\gamma$, the corresponding density profile reproduces the coreless density model:

$$
\rho(r)=1.5 \times 10^{5}\left(\frac{r}{1 \mathrm{pc}}\right)^{-1.8} M_{\odot} \mathrm{pc}^{-3}
$$

outside the core. This choice of normalizing constant gives a mass density at $1 \mathrm{pc}$ similar to what various authors have inferred (e.g., Oh et al. 2009) and implies a total mass in stars within this radius of $\sim 1.6 \times 10^{6} M_{\odot} \mathrm{pc}^{-3}$.

Assuming equal-mass stars of mass $m$ and an isotropic velocity distribution, the local two-body relaxation time is defined as (Spitzer 1987)

$$
t_{\mathrm{r}}=\frac{0.33 \sigma^{3}}{\rho m G^{2} \ln \Lambda},
$$

where $\ln \Lambda$ is the Coulomb logarithm and $\sigma$ is the isotropic velocity dispersion; the latter can be computed from Jeans's 

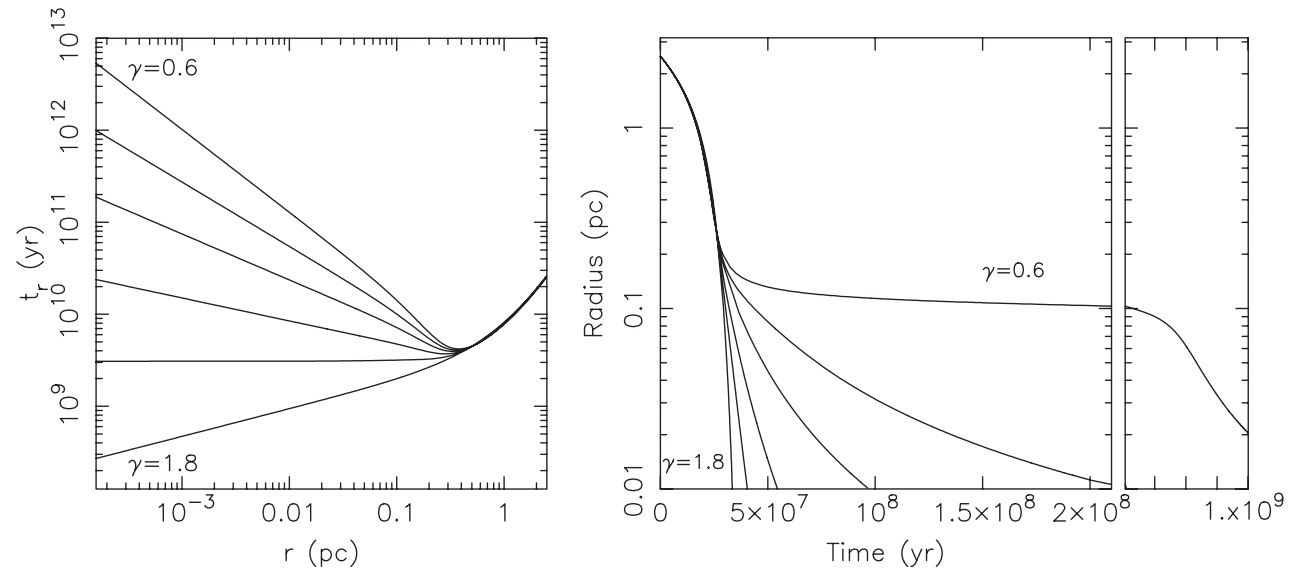

Figure 3. Left panel: relaxation time $t_{\mathrm{r}}$ vs. radius for models based on the density law of Equation (14). Right panel: orbital decay of a $2 \times 10^{3} M_{\odot}$ massive body starting from a radius of $2.5 \mathrm{pc}$. Here we used $\ln \Lambda=7$. In both panels, various values of the inner density slope $\gamma$ were considered: $(0.6,0.8,1,1.25,1.5,1.8)$.

equation,

$$
\rho(r) \sigma(r)^{2}=G \int_{r}^{\infty} d r^{\prime} r^{\prime-2}\left[M_{\bullet}+M_{\star}\left(<r^{\prime}\right)\right] \rho\left(r^{\prime}\right) .
$$

Here $M_{\bullet}$ is the mass of the central SMBH that we take to be $4 \times 10^{6} M_{\odot}$ (Ghez et al. 2008; Gillessen et al. 2009) and $M_{\star}(<r)$ is the total mass in stars within $r$. The total stellar mass contained within the $\mathrm{SMBH}$ influence radius $\left(r_{\mathrm{bh}} \approx 2.5 \mathrm{pc}\right)$ is $M_{\star}\left(<r_{\text {bh }}\right) \approx 10^{7} M_{\odot}$; assuming solar-mass stars, the two-body relaxation time at $r_{\mathrm{bh}}$ is $t_{\mathrm{r}}\left(r_{\mathrm{bh}}\right) \approx 2 \times 10^{10} \mathrm{yr}$.

\subsection{Circular Orbits}

The frictional acceleration on a point particle of mass $M$ and velocity $\boldsymbol{v}$ is (Chandrasekhar 1943)

$$
\boldsymbol{f}_{\mathrm{fr}}=-\frac{4 \pi G^{2} M \rho(r) F(<v, r) \ln \Lambda}{v^{3}} \boldsymbol{v},
$$

where $F(<v, r)$ is the fraction of stars at $r$ that are moving more slowly than $v$. This is the standard expression, derived by ignoring the velocity dependence of $\ln \Lambda$ when integrating over the field-star velocity distribution and setting the non-dominant terms to zero. As a result of these approximations, the frictional force is produced only by field stars with velocities less than $v$. Although Equation (18) was derived under the assumptions of an infinite and homogeneous background of stars, it has been shown to work reasonably well even for more general stellar distributions (White 1983; Lin \& Tremaine 1983; Weinberg 1986; Cora et al. 1997; Merritt 2006; Just et al. 2010).

For a massive particle initially located at $r_{\text {bh }}$ on a circular orbit, the inspiral time in the power-law density profile of Equation (14) with $\gamma=1.8$ (i.e., the coreless model) is

$$
\begin{aligned}
t_{\mathrm{fr}} \approx & 6 \times 10^{7} \mathrm{yr}\left(\frac{r}{2.5 \mathrm{pc}}\right)^{2}\left(\frac{\sigma}{100 \mathrm{~km} \mathrm{~s}^{-1}}\right)\left(\frac{1 \times 10^{3} M_{\odot}}{M}\right) \\
& \times\left(\frac{7}{\ln \Lambda}\right)
\end{aligned}
$$

independent of the mass of the field stars if $M \gg m$.

Figure 3 plots the relaxation time as a function of radius for the same model, assuming $\ln \Lambda=15, m=M_{\odot}$, and adopting different values for the inner density slope $\gamma$. It turns out that the isotropic distribution function corresponding to the adopted density law (14) becomes negative at certain energies for $\gamma \lesssim 0.6$. For this reason, we consider in the following only models with $\gamma \geqslant 0.6$. Figure 3 also shows the evolution of a $2 \times 10^{3} M_{\odot}$ black hole on a circular orbit starting from a galactocentric distance of $2.5 \mathrm{pc}$ and using $\ln \Lambda=7$. The orbit was numerically integrated by solving the system of first-order differential equations

$$
\dot{\boldsymbol{r}}=\boldsymbol{v}, \dot{\boldsymbol{v}}=-\nabla \phi+\boldsymbol{f}_{\mathrm{fr}}
$$

with $\phi(r)$ the total gravitational potential produced by the stars and the SMBH:

$$
\begin{aligned}
\phi(r)= & -\frac{G M_{\bullet}}{r}+\phi_{\star}(r)=-\frac{G M_{\bullet}}{r} \\
& +4 \pi G\left[\frac{1}{r} \int_{0}^{r} d r^{\prime} r^{\prime 2} \rho\left(\mathrm{r}^{\prime}\right)+\int_{r}^{\infty} d r^{\prime} r^{\prime} \rho\left(\mathrm{r}^{\prime}\right)\right] .
\end{aligned}
$$

The numerical integration was performed using a 7/8 order Runge-Kutta algorithm with a variable time step (Fehlberg 1968) in order to keep the relative error per step in energy, in the absence of dynamical friction, less than a specified value $\left(10^{-8}\right)$. When dynamical friction was included, we checked the integration accuracy through the quantity $E+E_{\mathrm{df}}$ with $E$ being the energy per unit mass and $E_{\mathrm{df}}$ the work done by dynamical friction along the trajectory. The accuracy in this case was of the same order as that found in integrations without dynamical friction. The function $F(<v, r)$ was evaluated using the expression (Szell et al. 2005):

$$
\begin{aligned}
F(<v, r)= & -\frac{1}{\rho} \int_{0}^{E} d \phi^{\prime} \frac{d \rho}{d \phi^{\prime}} \\
& \times\left\{1+\frac{2}{\pi}\left[\frac{v / \sqrt{2}}{\sqrt{\phi^{\prime}-E}}-\tan ^{-1}\left(\frac{v / \sqrt{2}}{\sqrt{\phi^{\prime}-E}}\right)\right]\right\},
\end{aligned}
$$

where $E=1 / 2\left(v^{2}+\phi(r)\right)$.

At all radii, the relaxation time is much longer than the time required for the massive particle to reach the core. What happens next depends on $\gamma$ : the orbital decay can essentially stall when $\gamma$ is small (i.e., $\sim 0.6$ ) or continue rapidly if $\gamma$ is larger.

The explanation of this behavior can be found in Figure 4 which plots the fraction of stars moving more slowly than the local circular velocity $v_{\text {circ }}(r)$ as a function of radius, for various values of $\gamma$. When $\gamma=0.6, F\left(<v_{\text {circ }}, r\right)$ approaches zero 


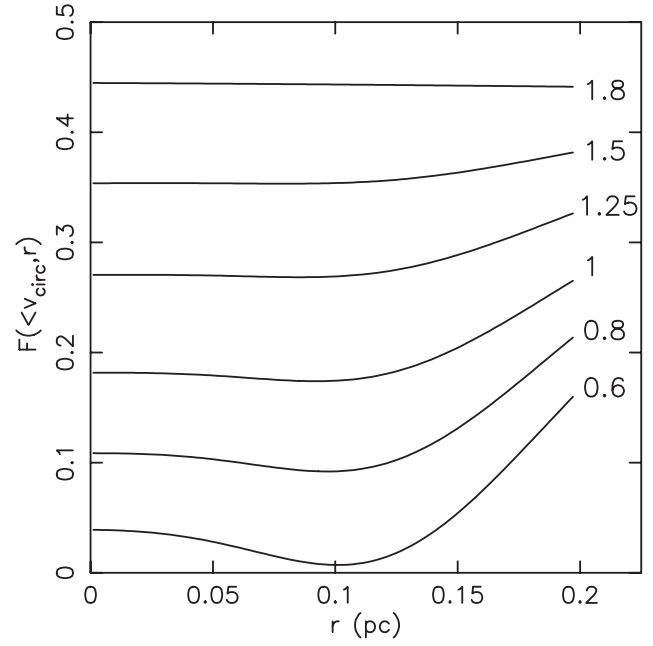

Figure 4. Fraction of stars $F\left(<v_{\text {circ }}, r\right)$ moving more slowly than the local circular velocity as a function of radius for $\gamma=(0.6,0.8,1,1.25,1.5,1.8)$. When $\gamma=0.6, F$ is close to zero for $r \approx 0.1 \mathrm{pc}$. Hence, the frictional force acting on a massive particle which moves on a circular orbit drops essentially to zero at this radius.

at $r_{\mathrm{st}} \sim 0.1 \mathrm{pc}$ and consequently the dynamical-friction force drops drastically at this radius (see Equation (18)). The stalling observed in the orbital evolution for this value of $\gamma$ is therefore a consequence of the lack of slowly moving stars in the core. However, the inspiral always continues into the very center since $F\left(<v_{\text {circ }}, r\right)>0$ everywhere.

For $\gamma \geqslant 0.6$, the time required for dynamical friction to bring a $10^{3} M_{\odot}$ black hole into the center, starting from a galactocentric distance of a few parsecs, is shorter than the twobody relaxation time evaluated at the $\mathrm{SMBH}$ influence radius $t_{\mathrm{r}}\left(r_{\mathrm{bh}}\right)$. On the other hand, the dynamical-friction force decreases with the mass of the inspiraling object, and for $M \lesssim 10^{2} M_{\odot}$ the infall timescale can significantly exceed a Hubble time. Merritt \& Szell (2006) found that $t_{\mathrm{r}}\left(r_{\mathrm{bh}}\right)$ is also approximately the timescale over which gravitational encounters change an initial density profile into the Bahcall-Wolf form, i.e., $\rho \propto r^{-1.75}$. We conclude that for a black hole of mass $M \geqslant 10^{3} M_{\odot}$, inspiral will occur in a mass profile that is almost independent of time. However, for $\gamma \sim 0.6$, the time required to reach a distance $\sim 0.01 \mathrm{pc}$ is still comparable with the local relaxation time. This will result in a substantial evolution of the stellar background during the orbital decay.

\subsection{Eccentric Orbits}

In the case of an isotropic distribution function $f(E)$ describing a power-law density profile around a $\mathrm{SMBH}$, if the gravitational potential produced by the stars is ignored (i.e., $\left.E \ll-G M_{\bullet} / r_{\text {bh }}\right)$, then

$f(E)=\frac{3-\gamma}{8} \sqrt{\frac{2}{\pi^{5}}} \frac{\Gamma(\gamma+1)}{\Gamma(\gamma-1 / 2)} \times \frac{M_{\bullet}}{m} \frac{\phi_{0}^{3 / 2}}{\left(G M_{\bullet}\right)^{3}}\left(\frac{|E|}{\phi_{0}}\right)^{\gamma-3 / 2}$,

with $\phi_{0}=G M_{\bullet} / r_{\text {bh }}$ (Merritt 2012). For $\gamma \leqslant 0.5, f(E)$ is undefined and so $\gamma \approx 0.5$ is the shallowest density profile consistent with an isotropic velocity distribution around a SMBH. In the case $\gamma=1.5$, Equation (23) shows that the distribution function is a constant $\left(f(E) \equiv f_{0}\right)$. If one writes

$$
\rho(r) F(<v, r)=\rho(r) \times \frac{1}{\rho(r)} 4 \pi \int_{0}^{v} d v_{\star} v_{\star}^{2} f_{0}=\frac{4}{3} \pi f_{0} v^{3}
$$

it can be immediately seen that the product $\rho(r) F(<v, r)$ in Equation (18) will be a function of $v$ only (e.g., Just et al. 2011). Under these circumstances, the coefficient of dynamical friction will have only a weak dependence on radius through the Coulomb logarithm. It can be shown that, in this case, the eccentricity of a massive body will remain unchanged during its motion, while dynamical friction will either circularize the orbit for $\gamma>1.5$ or make it more eccentric for $\gamma<1.5$ (Quinlan 1996; Gould \& Quillen 2003).

To evaluate the eccentricity evolution of a massive particle in response to Chandrasekhar's dynamical-friction formula, a numerical treatment is necessary. We therefore carried out numerical integrations of the set of differential equations (20) as described above, adopting as before Equations (14) and (21) for the (fixed) stellar potential.

Figure 5 shows the results for $M=2 \times 10^{3} M_{\odot}$. The massive particle was initially placed at $r=2.5 \mathrm{pc}$ with a tangential velocity of $\sim 0.36 v_{\text {circ }}$. With this initial configuration the body penetrates the inner core after few obits. Different values of the internal slope $\gamma$ ranging from 1.8 to 0.6 were adopted. As a proxy for the instantaneous orbital elements, we computed over each radial period the largest and the smallest distance from the origin (i.e., the SMBH) and defined these as the apoapsis $r_{\text {ap }}$ and periapsis $r_{\text {per }}$, respectively. The eccentricity and semimajor axis were then computed using the Keplerian expressions

$$
e=\frac{r_{\mathrm{ap}}-r_{\mathrm{per}}}{r_{\mathrm{ap}}+r_{\mathrm{per}}}, \quad a=\frac{r_{\mathrm{ap}}}{1+e} .
$$

The figure reveals a complex behavior of eccentricity on time. For $\gamma \leqslant 1.5$ we distinguish three regimes. In phase I, the eccentricity decreases (even for $\gamma \geqslant 1.5$ ). The duration of this phase is shorter for shallower profiles. After reaching a minimum, the eccentricity then increases rapidly with time (phase II). Finally, in phase III, the eccentricity either continues to increase, but more slowly than in phase II, or remains constant for $\gamma=1.5$.

This evolution can be understood by considering the changes of $r_{\text {ap }}$ and $r_{\text {per }}$ with time. In phase I, the black hole periapsis is close to the core radius, where the difference between the density models is small. As a consequence, the eccentricity evolution is nearly independent of $\gamma$ and the orbits circularize. In phase II, $r_{\text {per }}$ is well inside the core, where the smaller dynamical friction results in a rapid eccentricity increase. Finally, in phase III, the orbit lies entirely inside the core. As a consequence of the declining dynamical friction at $r_{\text {ap }}$ the eccentricity growth slows down. As predicted, for $\gamma=1.5$, the eccentricity remains unchanged in this phase.

These results show that, in the presence of a flat $(\gamma \lesssim 1)$ density profile, a second black hole found initially on an eccentric orbit can acquire very large eccentricities $(\lesssim 1)$ before entering the regime where relativistic effects become important. In Section 6.2, we discuss in more detail how very large eccentricities may modify the expectations for the gravitational wave $(\mathrm{GW})$ signal from massive black hole binaries for proposed space-based interferometers.

In the first phase, when the periapsis is still outside the core, the orbit evolves completely in the outer cusp $\left(\gamma_{e}=1.8\right)$. Evolution in this regime could lead to a rapid circularization before the black hole reaches the inner core. To quantify the amount of circularization in this phase we computed a further orbit in the model with $\gamma=0.8$, adopting initially a larger semimajor axis $(a \sim 10 \mathrm{pc})$ and a smaller eccentricity $(e=0.3)$. The results of this integration (Figure 6$)$ show that 

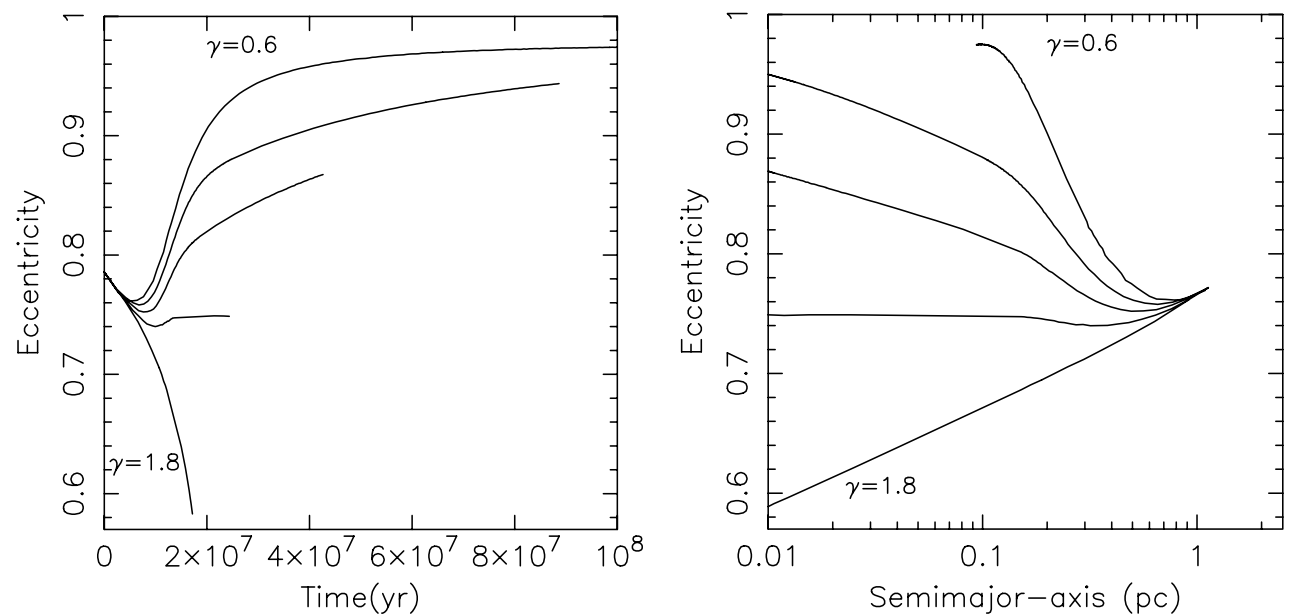

Figure 5. Left panel shows the time dependence of the orbital eccentricity of a $M=2 \times 10^{3} M_{\odot}$ black hole. In the right panel, the orbital evolution is shown in the eccentricity-semimajor axis plane. The inner cusp slopes are $\gamma=(0.6,1,1.25,1.5,1.8)$. Initial apoapsis and periapsis distances were 2.5 and 0.35 pc, respectively, and initial semimajor axis was $a=1.4 \mathrm{pc}$. The integrations terminated either when the semimajor axis of the black hole was $0.01 \mathrm{pc}$ or at $10^{8} \mathrm{yr}$ for $\gamma=0.6$.
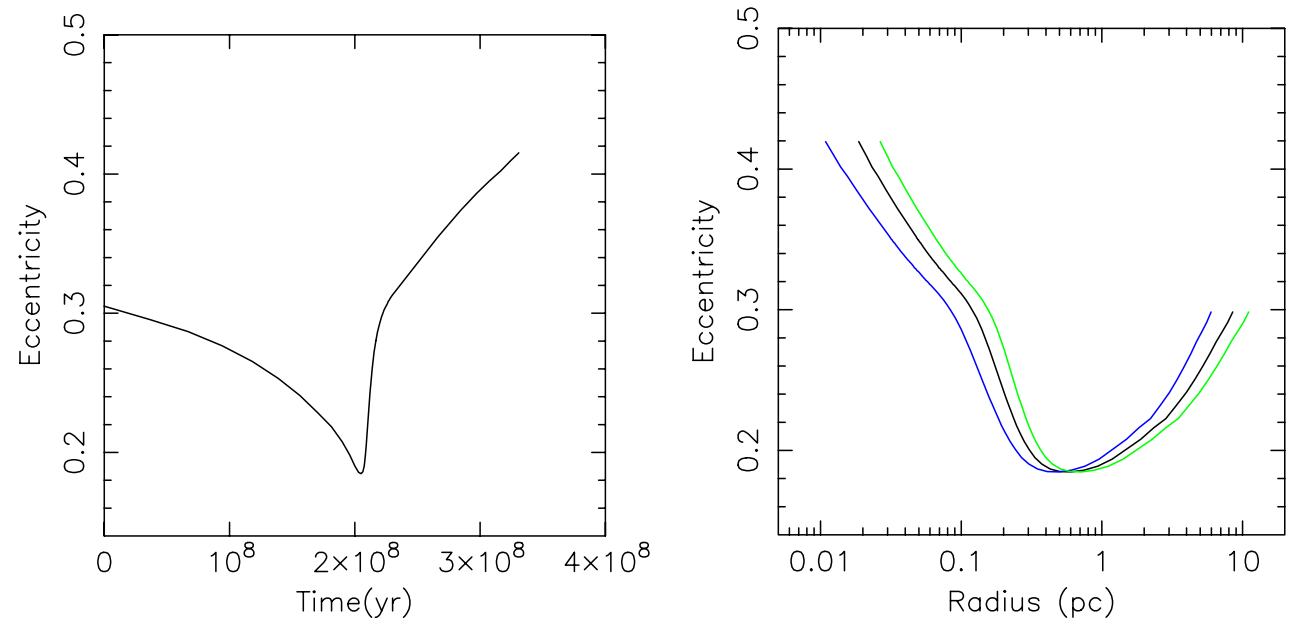

Figure 6. Left panel: eccentricity evolution for a $2 \times 10^{3} M_{\odot}$ black hole in a model with $\gamma=0.8$. The initial apoapsis and periapsis of the orbit are 12 and 7 pc, respectively, which give a semimajor axis $a \approx 9 \mathrm{pc}$. Right panel: eccentricity vs. semimajor axis (black line), apoapsis (green line), and periapsis (blue line).

(A color version of this figure is available in the online journal.)

the eccentricity reaches a minimum value, $e \approx 0.15$, and then increases rapidly reaching $e \approx 0.3$ at $r_{\text {per }}=0.1 \mathrm{pc}$. At the end of the integration the orbit retains therefore a substantial eccentricity $(\sim 0.4)$, even though it was almost circularized at the beginning of phase II.

\section{4. $N$-BODY SIMULATIONS}

The numerical integrations of Equation (18) presented above predict that a massive body that spirals into the center of a galaxy containing a SMBH, and a nuclear star cluster with flat $(\gamma \lesssim 0.6)$ density profile, will stall, at a radius that is roughly the core radius. Moreover, its eccentricity is expected to increase steeply once the orbital periapsis lies inside the core. Here we use $N$-body simulations to test these predictions.

\subsection{Initial Conditions and Numerical Method}

In order to generate equilibrium $\mathrm{N}$-body models of the GC region that extend self-consistently to the Sgr A* influence radius $\left(r_{\mathrm{bh}} \approx 2.5 \mathrm{pc}\right.$ ) we used the truncated mass model

$$
\rho(r)=\rho_{0}\left(\frac{r}{r_{0}}\right)^{-\gamma}\left[1+\left(\frac{r}{r_{0}}\right)^{\alpha}\right]^{\left(\gamma-\gamma_{e}\right) / \alpha} \zeta\left(r / r_{\mathrm{t}}\right)
$$

with truncation function

$$
\zeta(x)=\frac{2}{\operatorname{sech}(x)+\cosh (x)} .
$$

With this choice, the density falls off exponentially at large radii (i.e., $r>r_{\mathrm{t}}$ ), while for $r \ll r_{\mathrm{t}}$, where $\zeta(x) \approx 1-x^{4} / 8$, the model reproduces almost exactly the density of Equation (14). As above, we chose $r_{0}=0.3 \mathrm{pc}, \alpha=4, \gamma_{e}=1.8$, and $\rho_{0}=1.3 \times 10^{6} M_{\odot}$. Monte Carlo initial positions and velocities were then generated by numerically solving Equation (22); we stress that the equilibrium models so produced include selfconsistently the effects of the gravitational force from the stars. Figure 7 shows the truncated density profiles for different values of $\gamma$ and $r_{\mathrm{t}}=1.2 \mathrm{pc}$.

The initial conditions were evolved using the directsummation code $\phi$ GRAPE (Harfst et al. 2007) which uses a fourth-order Hermite integrator with a predictor-corrector scheme and hierarchical time steps. The performance and accuracy of the code depend both on the time step parameter $\eta$ and on the smoothing length $\epsilon$. In what follows, we set $\eta=0.01$ and $\epsilon=5 \times 10^{-4} \mathrm{pc}$. With these choices, energy conservation was typically of order $0.1 \%$ over the entire length of the integration. Most of the $N$-body integrations were carried out 


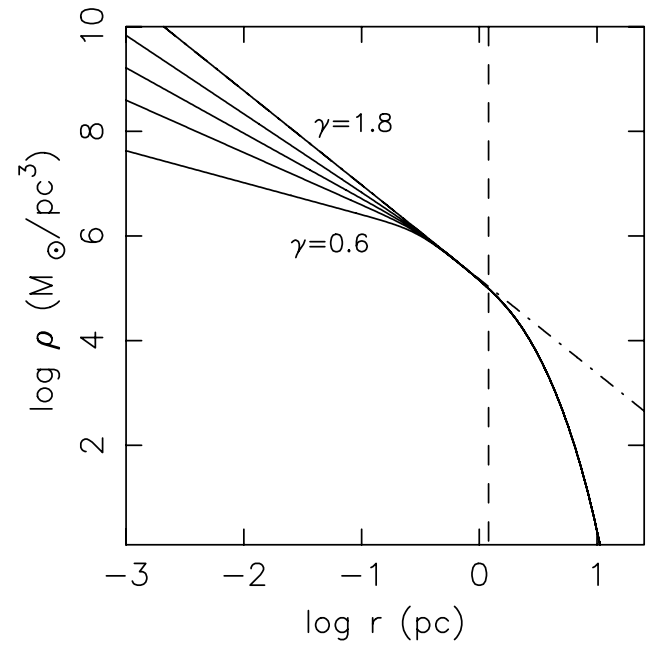

Figure 7. Density profiles of Equation (26) with $\gamma=(0.6,1,1.25,1.5,1.8)$, $r_{0}=0.3 \mathrm{pc}, \alpha=4$, and truncation radius $r_{\mathrm{t}}=1.2 \mathrm{pc}$ (vertical dashed line). The dash-dotted line gives the coreless model of Equation (15).

on the 32-node GRAPE cluster at the Rochester Institute of Technology. In addition, a few were carried out in serial mode using a TESLA C870 graphics processing unit with SAPPORO, a CUDA library that emulates double-precision force calculations on single-precision hardware (Gaburov et al. 2009).

Table 1 gives the parameters of the $N$-body models. The initial distance of the secondary black hole is given by $r_{\text {in }}$, while its initial orbital eccentricity is $e_{\text {in }}$. The quantity $r^{*}$ is the radius at which the initial mass in stars equals $M$, the mass of the second black hole. All of our $N$-body models had $r_{\text {in }}<r_{t}$, so that the orbital evolution is expected to be very similar to that in the corresponding non-truncated models. In order to study the dependence of the results on the secondary black hole mass we run simulations with a range of masses, $M=(2000,5000,10,000,50,000) M_{\odot}$. Two cases with nonzero initial eccentricities (runs G1 and G2, with $\left.e_{1}=0.54\right)$ were also considered.

\subsection{The Coulomb Logarithm}

In Table 1, we report the values of the Coulomb logarithm extracted from each $N$-body integration. The value of $\ln \Lambda$ was obtained by minimizing the quantity:

$$
\sum_{i=1}^{n}\left[r_{i}(t)-r^{\prime}(t, \ln \Lambda)\right]^{2},
$$

outside a galactocentric radius $r>0.3 \mathrm{pc}$. Here, $n$ is the number of $N$-body data points, $r_{i}(t)$ is the position of the black hole in the $N$-body simulation at time $t$, and $r^{\prime}(t)$ is its position at the same time evaluated by means of the Chandrasekhar's formula (18). Since analytical expressions are not available for the trajectory of an inspiraling black hole, in order to obtain the expected position $r^{\prime}(t)$ at any given time, we first solved numerically the equations of motion (20) and then built a spline interpolant from the results of the integration. This procedure was applied only in the part of the orbit outside the core, where Equation (18) is able to describe accurately the black hole orbit. In this way, unlike in most previous studies, we could obtain an estimate of the Coulomb logarithm without making any assumptions about the velocity distribution of the field stars (e.g., that it followed a Maxwellian distribution).

Our simulations do not show any obvious dependence of $\ln \Lambda$ on either the number of particles or on the initial eccentricity. We found an average value of $\ln \Lambda=6.5 \pm 0.2$, in essentially perfect agreement with the value reported by Spinnato et al. (2003): $\ln \Lambda=6.6 \pm 0.6$.

\subsection{Results \\ 4.3.1. Circular Orbits}

The first simulations we performed consisted in evolving the massive body on a circular orbit with initial radius 0.1 pc (i.e., smaller than the stalling radius when $\gamma \lesssim 0.6)$ and for a time corresponding approximately to 300 orbits (i.e., $\sim 4 \times 10^{5} \mathrm{yr}$ at this distance). We used $N=130,000, M=5000 M_{\odot}$, and $\gamma=(0.6,1,1.5,1.8)$. We also implemented a high-resolution simulation with $N=500,000$ for the model with $\gamma=0.6$. As in most of the longer simulations of Table 1 , the truncation radius was $r_{\mathrm{t}}=1.2 \mathrm{pc}$. These shorter integrations allowed us to study dynamical friction, while limiting the deviations of the models from their initial configuration that was found to occur on longer timescales as a result of two-body relaxation and perturbations from the massive object (see below). The eccentricity of the orbit remained small during these integrations $(e \lesssim 0.1)$.

Figure 8 shows the time evolution of the semimajor axis of the orbits and the rate of orbital decay $s=-d a / d t$ as a function of $\gamma$. The agreement with the decay rate computed using Chandrasekhar's formula (18) (open squares) is good. For $\gamma=0.6$, there is not any significant evolution of the orbit in the considered interval of time and, consequently, $s \approx 0$.

A similar conclusion is implied by Figure 9 which shows the trajectory of a $2000 M_{\odot}$ black hole in model D, a longer integration with $N=130,000$ and $\gamma=0.6$. Initially, the black

Table 1

Initial Models Parameters and Coulomb Logarithm Estimates

\begin{tabular}{|c|c|c|c|c|c|c|c|c|c|}
\hline Model & $\gamma$ & $\begin{array}{l}N \\
\mathrm{k}\end{array}$ & $\begin{array}{c}r_{\mathrm{t}} \\
(\mathrm{pc})\end{array}$ & $\begin{array}{c}M \\
\left(10^{3} M_{\odot}\right)\end{array}$ & $\begin{array}{c}m \\
\left(M_{\odot}\right)\end{array}$ & $e_{\text {in }}$ & $\begin{array}{c}r_{\text {in }} \\
(\mathrm{pc})\end{array}$ & $\begin{array}{c}r^{*} \\
(\mathrm{pc})\end{array}$ & $\ln \Lambda$ \\
\hline A1 & 0.6 & 230 & 1.2 & 5 & 22 & 0 & 1 & 0.07 & 6.7 \\
\hline A2 & 0.6 & 130 & 1.2 & 5 & 38 & 0 & 1 & 0.07 & 6.6 \\
\hline B1 & 0.8 & 230 & 1.2 & 5 & 22 & 0 & 1 & 0.06 & 6.9 \\
\hline B2 & 0.8 & 130 & 1.2 & 5 & 38 & 0 & 1 & 0.06 & 6.9 \\
\hline $\mathrm{C}$ & 0.6 & 80 & 0.6 & 5 & 26 & 0 & 0.5 & 0.07 & 6.3 \\
\hline D & 0.6 & 130 & 1.2 & 2 & 38 & 0 & 0.3 & 0.05 & $\ldots$ \\
\hline $\mathrm{E}$ & 0.6 & 130 & 1.2 & 10 & 38 & 0 & 1 & 0.10 & 6.4 \\
\hline $\mathrm{F}$ & 0.6 & 130 & 1.2 & 50 & 38 & 0 & 1 & 0.18 & 4.8 \\
\hline G1 & 0.6 & 200 & 1.2 & 5 & 25 & 0.54 & 1 & 0.07 & 6.9 \\
\hline G2 & 0.6 & 100 & 1.2 & 5 & 50 & 0.54 & 1 & 0.07 & 6.9 \\
\hline
\end{tabular}



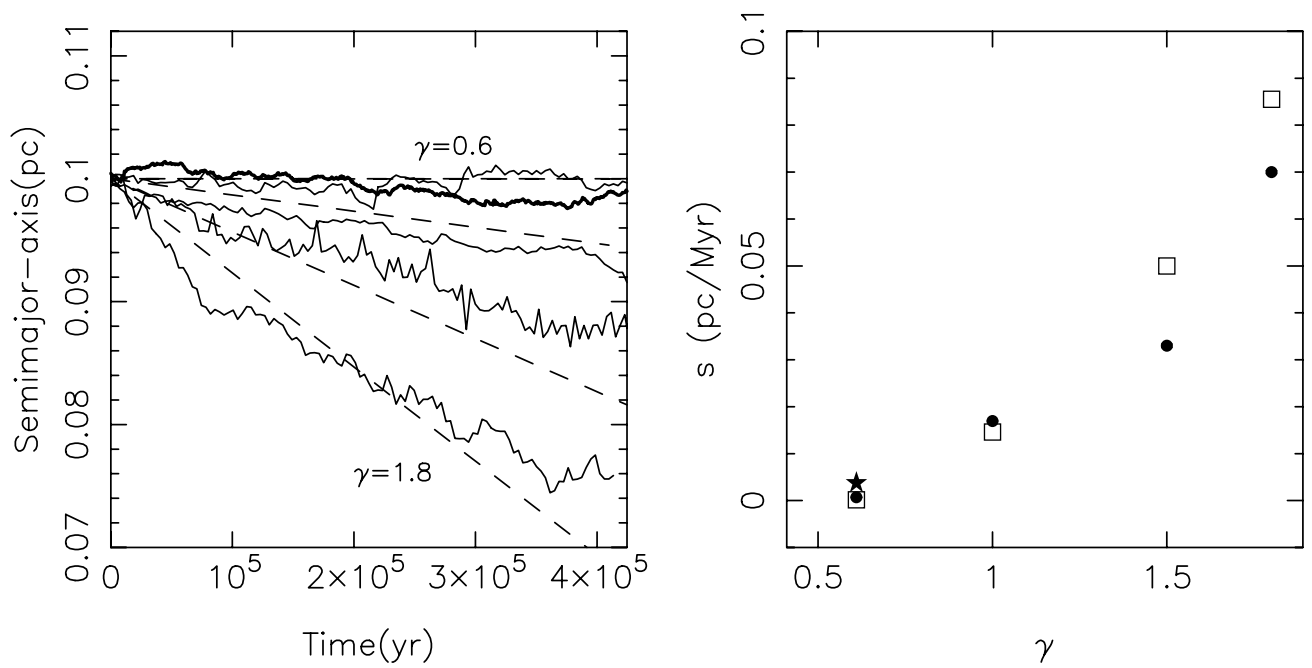

Figure 8. Left panel: evolution of the semimajor axis for a $5000 M_{\odot}$ black hole in the short $N$-body integrations, for different values of the central density slope (from top to bottom, $\gamma=0.6,1,1.5,1.8$ ). The thicker line is from the high- $N$ integration, with $N=500,000$ and $\gamma=0.6$. Dashed lines are predictions from Chandrasekhar's formula (18) using $\ln \Lambda=6.6$. For $\gamma=0.6$ there is no significant evolution of the orbit in the considered interval of time. Right panel: orbital inspiral rates $s=-d a / d t$ computed for the simulations displayed on the left panel as a function of $\gamma$ (filled circles). Open squares give the predictions from Chandrasekhar's formula. The star symbol is the decay rate computed from the high-resolution run $(N=500,000$ and $\gamma=0.6)$.

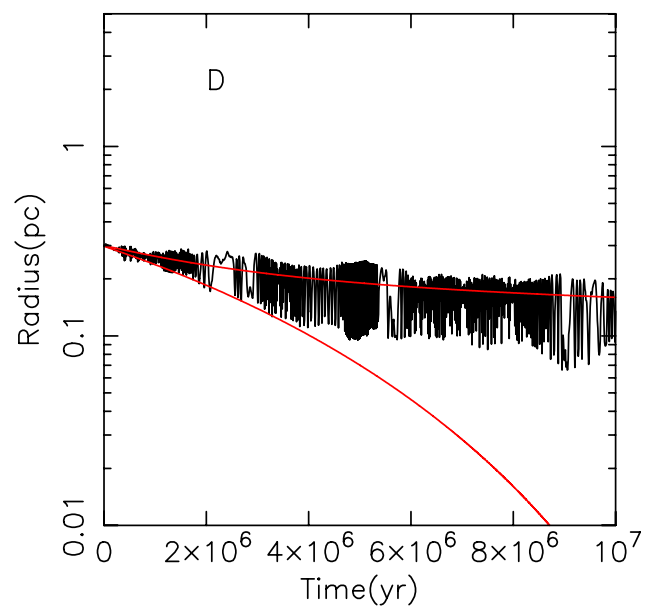

Figure 9. Trajectory of a $2000 M_{\odot}$ black hole into a core with $\gamma=0.6$ (model $D$ ). The top red line is the theoretical prediction obtained from Chandrasekhar's formula (18) using $\ln \Lambda=6.6$. The bottom red curve shows the predicted inspiral in a $\gamma=1.8$ cusp.

(A color version of this figure is available in the online journal.)

hole sinks rapidly to the center, reaching $\sim r_{\mathrm{st}}$ in $\sim 3 \mathrm{Myr}$. As the inspiral progresses, the orbit becomes more eccentric $(e \approx 0.3$ at $4 \mathrm{Myr})$. At later times $(\gtrsim 4 \mathrm{Myr})$, the orbit shows no sign of further decay, oscillating in radius between $\sim 0.1$ and $\sim 0.2 \mathrm{pc}$. The orbital eccentricity remains almost constant in this phase.

These findings, obtained for a flattened density cusp around a $\mathrm{SMBH}$, seem to confirm the theoretical predictions made above: (1) dynamical friction "vanishes" within $r_{\mathrm{st}} \approx 0.15 \mathrm{pc}$ and (2) the orbital eccentricity of an infalling body increases with time.

However, in any $N$-body simulation, stars are continuously scattered by gravitational encounters with other stars, with the result that the initially empty phase-space region responsible for the vanishing dynamical-friction force will gradually be filled. In addition, due in part to the low central density of our GC models when $\gamma$ is small, the radius at which the cumulative mass in stars becomes comparable to that of the inspiraling black hole can be of order $r_{\mathrm{st}}$, even for relatively small $M$ (see Table 1). $N$-body simulations have shown that, in these circumstances, the orbit deviates from the theoretical prediction of the Chandrasekhar's formula as a consequence of perturbations induced by the infalling black hole on the inner cusp (Baumgardt et al. 2006; Löckmann \& Baumgardt 2008). Finally, it is not clear whether the approximations made in deriving Equation (18), which was the basis for the red lines plotted in Figure 9, are reasonable, or how large might be the frictional force from fast-moving stars that populate the lowdensity core. In fact, as we now demonstrate, these additional effects have a substantial influence on the long-term evolution of the black hole orbit.

Figure 10 shows the trajectory of the black hole for some of the $N$-body integrations from Table 1 and compares them to the evolution predicted by Chandrasekhar's formula (18) (upper green curves). (In the upper panels, the comparison is displayed only for the higher resolution runs, i.e., models A1 and B1.) Although the agreement with the theoretical prediction appears fairly good, at least for $M=5000 M_{\odot}$, when $\gamma=0.6$, the $\mathrm{N}$-body integrations reveal a faster decay than predicted. Either some of the frictional force must come from stars with velocities $v_{\star}>v$, or the background stellar distribution is changing during the inspiral (or both). These two possibilities are investigated in what follows.

Dynamical friction from fast-moving stars. Equation (18) was derived under standard approximations that ignore the contribution from non-dominant terms and the velocity dependence of $\ln \Lambda$. Although these approximations are reasonable when there is a large fraction of stars with low velocities (i.e., $v_{\star}<v$ ), it is unclear whether they can be applied to a region populated mostly by stars moving faster than the black hole.

Without these assumptions, the instantaneous dynamicalfriction acceleration becomes (Chandrasekhar 1943)

$$
\begin{aligned}
\boldsymbol{f}_{\mathrm{fr}}= & -4 \pi G^{2} M \rho(r) \frac{\boldsymbol{v}}{v^{3}} \int_{0}^{\sqrt{-2 \phi(r)}} d v_{\star} 4 \pi f\left(v_{\star}\right) v_{\star}^{2} \\
& \times \frac{1}{8 v_{\star}} \int_{\left|v-v_{\star}\right|}^{v+v_{\star}} d V\left(1+\frac{v^{2}-v_{\star}^{2}}{V^{2}}\right) \ln \left(1+\frac{p_{\max }^{2} V^{4}}{G^{2} M^{2}}\right),
\end{aligned}
$$



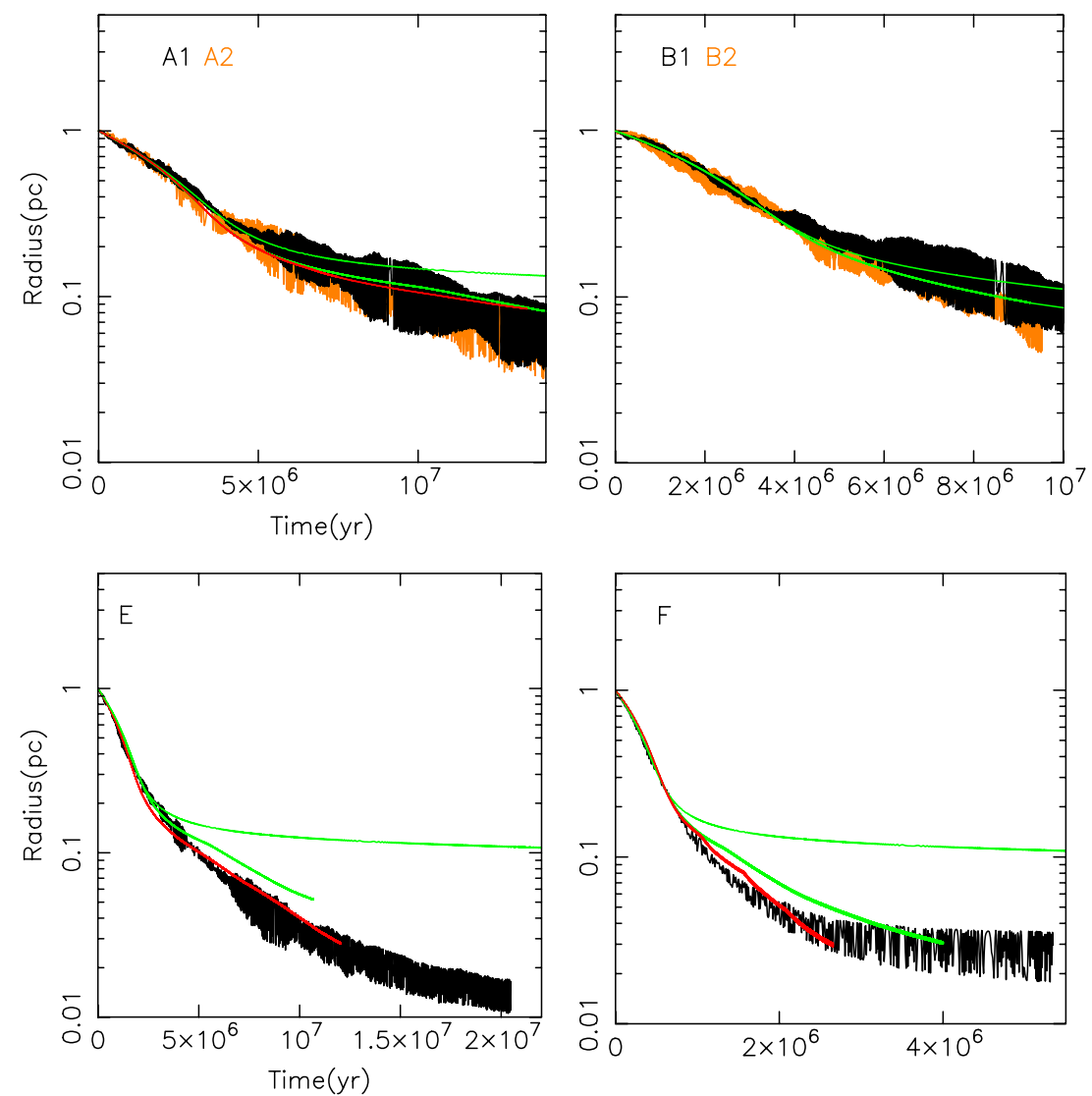

Figure 10. Orbital evolution of the second black hole in models A1, A2, B1, B2, E, and F. Solid green lines show predictions assuming a fixed background of stars. Upper green curves are obtained by using the standard Chandrasekhar's formula (i.e., Equation (18)), while lower green curves give the orbital decay computed using Equation (29) with $p_{\max }=0.5 \mathrm{pc}$. Red lines were obtained with Equation (29) but allowing $f\left(v_{\star}\right)$ and $\rho(r)$ to change according to the evolution of the $N$-body system.

where $f\left(v_{\star}\right)$ is the velocity distribution of field stars and $p_{\max }$ is the effective, maximum value of the impact parameter. In this more accurate treatment, some of the dynamical-friction force is due to stars moving more rapidly than the massive particle (Chandrasekhar 1943; White 1949; Merritt 2001). If the condition $p_{\max } V^{2} / G M \gg 1$ is satisfied, the frictional force can be approximated as (Chandrasekhar 1943; Equation (30))

$$
\begin{aligned}
\boldsymbol{f}_{\mathrm{fr}} \approx & f_{\mathrm{fr}}^{\left(v_{\star}<v\right)}+\boldsymbol{f}_{\mathrm{fr}}^{\left(v_{\star}>v\right)}=-4 \pi G^{2} M \rho(r) \frac{\boldsymbol{v}}{v^{3}} \\
& \times\left(\int_{0}^{v} d v_{\star} 4 \pi f\left(v_{\star}\right) v_{\star}^{2} \ln \left[\frac{p_{\max }}{G M}\left(v^{2}-v_{\star}^{2}\right)\right]\right. \\
& \left.+\int_{v}^{\sqrt{-2 \phi(r)}} d v_{\star} 4 \pi f\left(v_{\star}\right) v_{\star}^{2}\left[\ln \left(\frac{v_{\star}+v}{v_{\star}-v}\right)-2 \frac{v}{v_{\star}}\right]\right) .
\end{aligned}
$$

Inside $r_{\mathrm{st}}$, dynamical friction is produced mostly by stars with $v_{\star}>v$ and the first term in the integral becomes negligible. This shows the weak dependence of the frictional deceleration inside the core on $p_{\max }$.

Adopting Equation (29), with $p_{\max }=0.5 \mathrm{pc}$, for the frictional force that appears in the equations of motion (20), we obtained the lower green curves in Figure 10, which show much better agreement with the $N$-body results. Evidently, the standard expression for dynamical friction, Equation (18), is inadequate to describe the orbital evolution of a massive body at the GC in the case that the density profile of the nuclear star cluster is shallow. This is apparently a consequence of neglecting the non-dominant terms, and not, for instance, of the assumed independence of the Coulomb logarithm on the fieldstar velocity distribution. For models A1 and A2, Lagrangian radii showed essentially no evolution, indicating the absence of any significant change in the stellar distribution induced by the second black hole. We conclude that (at least) some of the drag within $r_{\text {st }}$ is due to field stars with $v_{\star}>v$. The red lines in Figure 10 were derived from Equation (29) but using a timedependent distribution function $f\left(v_{\star}, t\right)$ extracted (at time $t$ ) from the $N$-body models (see below). For models A1 and A2, the red curves agree exceptionally well with the $N$-body results and they essentially match the results of the semianalytical integration that takes into account the friction from fast-moving stars. We conclude that for these runs it would be appropriate to ignore the influence of the second black hole on the stellar distribution.

In the left panel of Figure 11 we plot the fraction of the dynamical-friction force that is predicted, by Equation (29), to come from stars with $v_{\star}>v$, for different values of the inner cusp slope and at different radii. In the right panel of the figure, the total frictional deceleration in our models is given in units of the frictional force computed under the assumption of a Maxwellian distribution of velocities:

$$
f_{\mathbf{f r}}^{\prime}=\frac{-4 \pi G^{2} M \rho(r) \ln \Lambda}{v^{3}} \boldsymbol{v}\left[\operatorname{erf}(X)-\frac{2 X}{\sqrt{\pi}} e^{-X^{2}}\right],
$$

with $X=v / \sqrt{2} \sigma$. Clearly, this equation, often used in the past to describe the orbital evolution of a massive object into the GC, overestimates the frictional drag within $r \lesssim 0.2$ pc for $\gamma \lesssim 1$. 

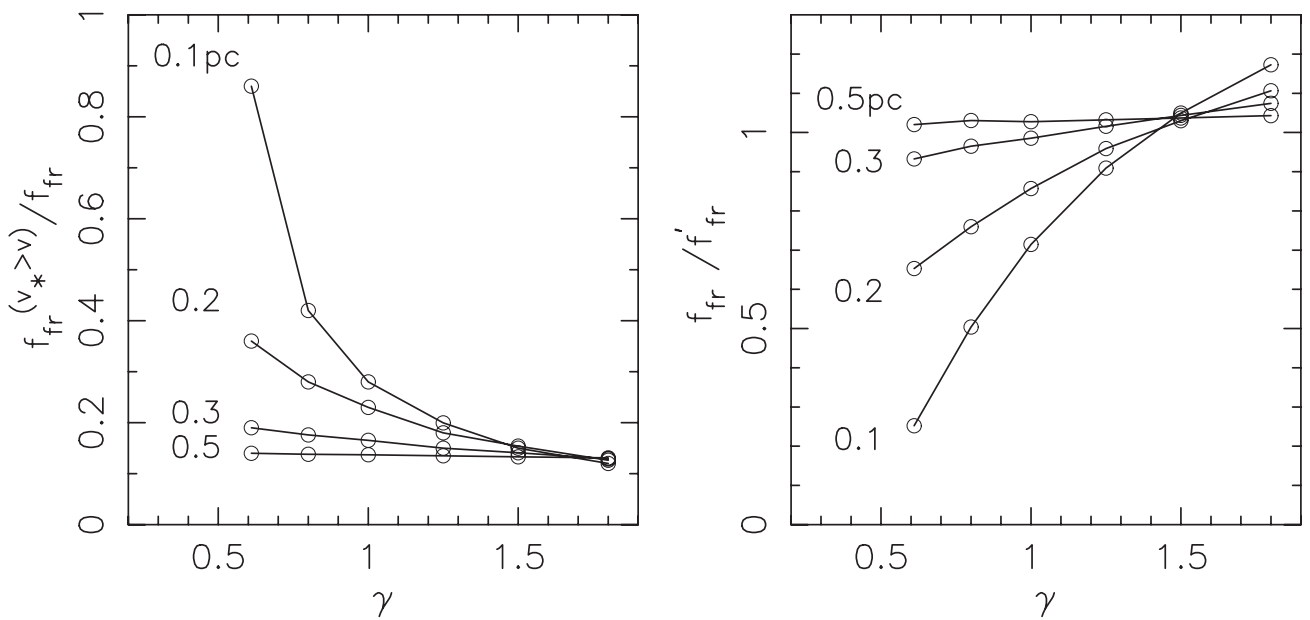

Figure 11. Left panel: fraction of the dynamical-friction force that is predicted to come from stars with $v_{\star}>v$ as a function of $\gamma$, at different galactocentric radii: $r=0.1,0.2,0.3$, and $0.6 \mathrm{pc}$. Equation (29) was used to compute these curves. When $\gamma=0.6$, dynamical friction at small radii comes only from stars with $v_{\star}>v$. As either $\gamma$ or $r$ increase, the contribution from fast-moving stars decreases. Right panel: total dynamical-friction force in units of the frictional deceleration computed assuming a Maxwellian distribution of velocities. The frictional force produced by stars with $v_{\star}>v$ in the flattened cusp (i.e., $\gamma=0.6$ and $r \lesssim 0.2$ pc) is much smaller than that obtained under the simple assumption of thermal distribution of velocities. In both panels, we adopted $p_{\max }=0.5 \mathrm{pc}$ and $M=1000 M_{\odot}$. In the right panel, we used $\ln \Lambda=6.6$ to solve Equation (31).
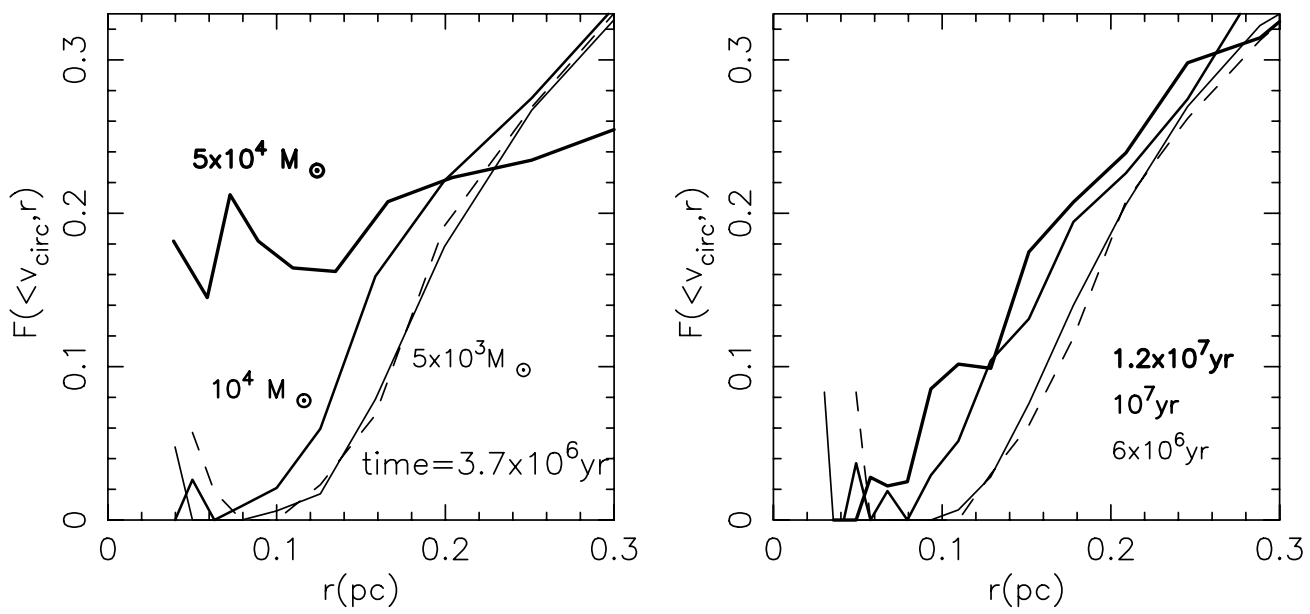

Figure 12. Left panel: fraction of stars with velocities less than the local circular velocity $F\left(<v_{\text {circ }}, r\right)$ as a function of radius, at the same time $\left(3 \times 10^{6}\right.$ yr $)$ for models A2 $\left(M=5000 M_{\odot}\right), \mathrm{E}\left(10,000 M_{\odot}\right)$, and $\mathrm{F}\left(50,000 M_{\odot}\right)$. The dashed curve corresponds to the initial configuration. The larger the mass of the black hole the faster the changes of the model in velocity space. Right panel: $F\left(<v_{\text {circ }}, r\right)$ as a function of radius for model A1 at different times. Due to two-body relaxation, stars are scattered toward low velocities and the hole in phase space that characterized the initial configuration is gradually filled up.

Influence of the second black hole on the field-star distribution. For larger masses of the infalling body, i.e., $M \gtrsim$ $10,000 M_{\odot}$, the perturbations which it induces in the background system introduce a complex time dependence of the phase-space distribution. During the orbital inspiral, the black hole scatters stars into the inner cusp; consequently, once it reaches $\sim r_{\text {st }}$, it will "see" stars with $v_{\star}<v$ that contribute to the frictional acceleration from that point on.

In order to test Chandrasekhar's formulae under these circumstances, the black hole equations of motion were integrated in a time-varying potential whose properties were varied over time in a way designed to mimic the evolving $N$-body models. In more detail, the density of the $\mathrm{N}$-body model was computed at fixed intervals of time by binning particles in concentric logarithmically spaced shells. At the same time the velocity distribution of field stars was obtained directly from the $N$-body model. Finally, the black hole equations of motion were numerically integrated as described in Section (3.1) using expression (29). In this way, we were able to approximately account for the back reaction of the second black hole on the stellar distribution. It is worth noting that, even with this more sophisticated approach, two relevant assumptions are retained: (1) any induced deviation of the models from isotropy is neglected; (2) the black hole is assumed to move always on a circular orbit, while the $N$-body simulations clearly show an increase of the orbital eccentricity with time. The red curves of Figure 10, obtained through this numerical procedure, show that even when the galactic nucleus is rapidly deviating from its initial configuration, Chandrasekhar's theory can still accurately reproduce the $N$-body results if the changes in the stellar distribution are taken into account and the fast-moving stars are included when computing the frictional force.

In Figure 12 we show the evolution induced by the second black hole in the velocity distribution of the model, by plotting the function $F\left(<v_{\text {circ }}, r\right)$ at the same time $\left(3 \times 10^{6} \mathrm{yr}\right)$ for different masses (left panel). In addition, we show how $F\left(<v_{\text {circ }}, r\right)$, for $M=5000 M_{\odot}$, evolves as a function of time (right panel). In this latter case, two-body relaxation causes the diffusion of stars at low velocities and the stalling radius is shifted from the initial $\approx 0.1 \mathrm{pc}$ to $\approx 0.05 \mathrm{pc}$ by the end of the 

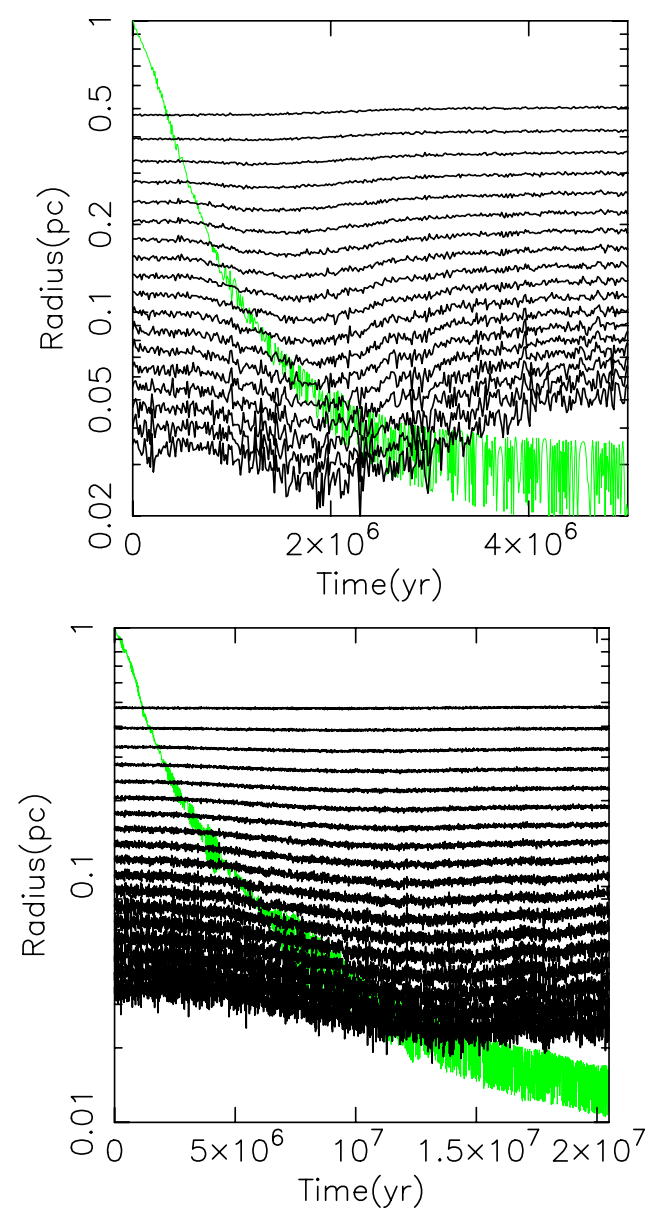

Figure 13. Lagrangian radii evolution of models F (upper panel) and E (lower panel). Green curves show the position of the massive body.

(A color version of this figure is available in the online journal.)

simulation. We note that-in a real galaxy with much larger $N$-this effect would be essentially absent.

Figure 13 illustrates the changes in the configuration-space density for models $\mathrm{E}$ and $\mathrm{F}$ via the time evolution of their Lagrangian radii. The time evolution of models $\mathrm{E}$ and $\mathrm{F}$ is remarkable: in model $\mathrm{F}$, the perturbations on the stellar distribution are initially so large that the core fills up during the first $\sim 2 \times 10^{6} \mathrm{yr}$. At this point, the black hole, at a galactocentric distance of $\sim 0.05 \mathrm{pc}$, starts to carve out the inner region, destroying the cusp that it created before. The final model has a core of size $\sim 0.2 \mathrm{pc}$ and the internal slope is $\gamma \lesssim 0.5$. However its density is, everywhere within $1 \mathrm{pc}$, smaller than that of the initial model as a consequence of displacement of stars from the cusp. A qualitatively similar evolution was found in model E. Figure 14 shows the induced evolution of the density profile for runs $\mathrm{E}$ and $\mathrm{F}$ as well as the time variation of the anisotropy parameter, defined as

$$
\beta=1-\sigma_{t}^{2} / \sigma_{r}^{2}
$$

with $\sigma_{t}$ and $\sigma_{r}$ tangential and radial velocity dispersions, respectively.

In summary, a straightforward interpretation of our $N$-body results is that Equation (18) reproduces remarkably well the real decay rate of a massive object into the GC only until it reaches the stalling radius. In the subsequent evolution, the orbital decay slows down as a consequence of the lack of slow-moving stars in the inner galactic nucleus (see Figures 8 and 9), but it never drops to zero, due apparently to the frictional force generated by stars moving faster than the inspiraling black hole (Figures 10 and 11).

A massive body of mass $M \approx 1000 M_{\odot}$, starting from distances of order $r_{\mathrm{bh}}$, will reach a galactocentric radius $\sim 0.01 \mathrm{pc}$ in $\sim 10^{8}$ yr. For larger masses (i.e., $M \gtrsim 10,000 M_{\odot}$ ), during the inspiral, the black hole enhances the diffusion of stars into the phase-space region that was initially nearly empty (Figures 12 and 13). During the stalling phase a low-density core is rapidly regenerated by the second black hole as it displaced stars from the cusp. Note that in our models the stalling distance is about 10 times larger than that found in previous works that assumed a collisionally relaxed, steeply rising density profile around the central black hole (e.g., Baumgardt et al. 2006; Löckmann \& Baumgardt 2008).

We note in passing that the background stars have orbital periods similar to that of the massive body. It is conceivable that correlations may be induced by the massive body in the orbital elements of the stars that will change the evolution significantly away from that produced by an uncorrelated background. On the other hand, two-body relaxation in the $N$-body models will tend to de-correlate the background response, leading, perhaps, to a better correspondence with the predictions of Chandrasekhar's theory.

\subsubsection{Eccentric Orbits}

In this section, we investigate the rate of change of the orbital eccentricity as a consequence of dynamical friction. We devised two simulations that differ only in the number of particles: 200,000 and 100,000. We refer to these simulations as runs G1 and $\mathrm{G} 2$, respectively (see Table 1 ); both have $\gamma=0.6$. The black hole was initially placed at a radius of $r_{\text {in }}=1 \mathrm{pc}$ on an eccentric orbit with $e_{\text {in }}=0.54$. As discussed earlier (Section 3.2), when the orbital periapsis lies within the core, the orbit is expected to become more eccentric as a consequence of the declining frictional force in this region.

Figure 15 shows the evolution of the eccentricity and semimajor axis of the orbit as a function of time, demonstrating that, at least qualitatively, Chandrasekhar's theory reproduces the evolution. Although the eccentricity undergoes significant fluctuations, it evidently drifts toward larger values with time. This behavior is quite robust showing a negligible $N$-dependence.

It is generally assumed that dynamical friction, in power-law density models with an isotropic velocity distribution, would circularize the orbit of an infalling body (see for instance Baumgardt et al. 2006). Our $N$-body simulations demonstrate that in models characterized by a flat density profile and a central $\mathrm{SMBH}$, the eccentricity can instead be an increasing function of time.

\section{GRAVITATIONAL WAKE}

An alternate way to look at dynamical friction is in terms of the acceleration produced by the overdensity of stars that accumulate behind the massive body - the "gravitational wake" (Danby \& Camm 1957; Marochnik 1968; Mulder 1983). The expression for the response wake in a homogeneous medium is given for arbitrary spherical density distribution in Weinberg (1986). The existence of a wake has rarely been confirmed in $N$ body simulations; an isolated example is provided by Weinberg \& Katz (2002, see also Weinberg \& Katz 2007) who show the wake induced in a dark matter halo by a stellar bar. Other examples include Weinberg (1989), Hernquist \& Weinberg (1989), and Vesperini \& Weinberg (2000). 

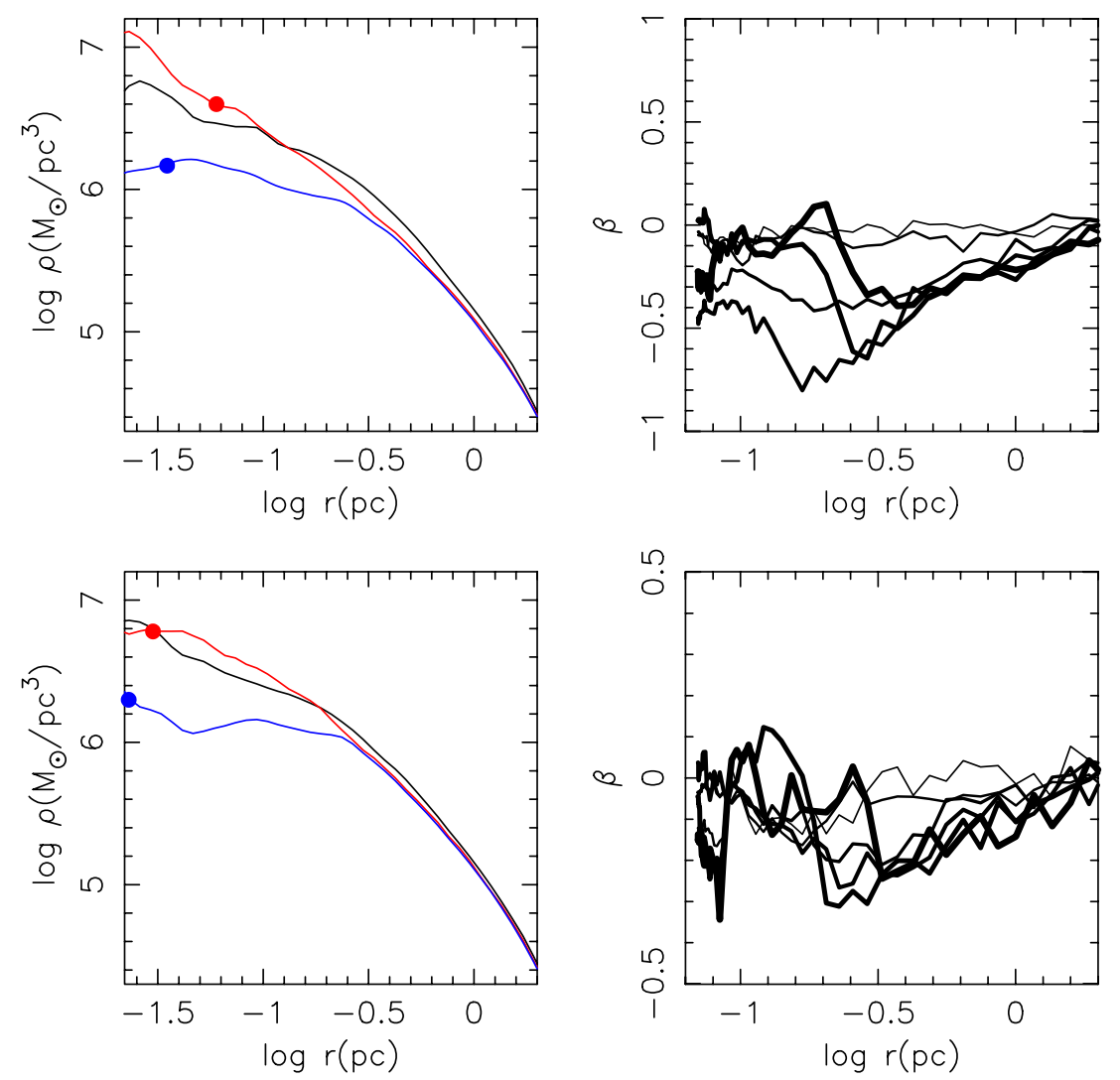

Figure 14. Left panels: density profile evolution in run F (upper panel) and $\mathrm{E}$ (lower panel). The black curve corresponds to the initial model; the red line is obtained at time $10^{7} \mathrm{yr}$ for run $\mathrm{E}$ and at $2 \times 10^{6} \mathrm{yr}$ for run $\mathrm{F}$, while the blue lines are the density profile of the final models, after the secondary black hole has stalled carving out a deficiency of stars in the inner regions. Filled circles indicate the position of the inspiraling. Right panels: evolution of the anisotropy parameter in the models. Line thickness increases with time. As the black hole spirals in, it induces tangential anisotropy in the background system.

(A color version of this figure is available in the online journal.)

We searched for the wake in our $N$-body simulations by computing the relative overdensity at each radius along the orbit of the second black hole. The $N$-body models were first rotated in such a way that the second black hole was situated at $y=z=0$ with $v_{z}=0$ and $v_{y}>0$. The density at any position was then estimated using a Gaussian kernel with radially varying smoothing length. Figure 16 shows the results in runs $\mathrm{A} 1, \mathrm{E}$, and $\mathrm{F}$ as a function of the azimuthal angle $\theta$ at different radii and for different values of $M$. In the figure, the black hole lies at $\theta=0$ with $\dot{\theta}>0$ and the average density is defined as $(1 / 2 \pi) \int_{-\pi}^{\pi} d \theta \rho(\theta)$ : outside the core $(r \gtrsim 0.3 \mathrm{pc})$, the peak in the overdensity lies at $-20<\theta \lesssim 0^{\circ}$, independent of $M$, and the amplitude of the overdensity increases with black hole mass, as expected. The wake is therefore always just behind the massive body in this phase. When $r \lesssim$ $0.3 \mathrm{pc}$, for $M=5000-10,000 M_{\odot}$, the density enhancement is reduced but its position remains essentially unchanged. The reduced amplitude of the wake inside the core explains why the frictional force is greatly suppressed in these regions. For larger masses, the angular dependence of the overdensity in this phase is more complex, revealing, in some cases, two distinct peaks. During this phase, the mass distribution is affected by gravitational scattering from the massive body. Finally, when the black hole is well inside the core, the density maximum is seen to lie at large angular separations $\left(\theta \lesssim-100^{\circ}\right)$ from the black hole. Indeed, a density "hole," with amplitude approximately proportional to $M$, is apparently induced by the black hole at roughly its position during the stalling phase.
Figure 17 shows two-dimensional contour maps of the overdensity for run $\mathrm{E}\left(M=5 \times 10^{4}\right)$. The radial extension of the wake (with respect to the galaxy center) does not change greatly over time, but one can clearly see how the location of the density maximum shifts, and a density gap is apparently created near the black hole position during the stalling phase.

To more clearly illustrate how the location of the gravitational wake with respect to the second black hole evolves, we plot in Figure 18 the angular position of the maximum as a function of the black hole galactocentric radius. Outside the core (i.e., $r>0.3 \mathrm{pc}$ ) the wake is located at small (negative) angles, causing the initial rapid inspiral. Once the black hole starts to modify the background of stars the wake becomes more difficult to track. This causes the large oscillations seen in the relative position of the wake and in turn explains why such oscillations occur earlier for larger masses of the inspiraling object.

\section{DISCUSSION}

In this paper, we presented $N$-body simulations of the inspiral of a massive body into the GC. Our models of the Milky Way nuclear star cluster were motivated by recent observations that suggest a relatively low density of stars inside the SMBH influence radius. Such models are characterized by a zero or near-zero phase-space density at low energies. Under the standard approximation, in which the frictional force from fastmoving stars is ignored, a second black hole that sinks toward the center under the influence of dynamical friction would stall at a distance of roughly $1 / 2$ the core radius, or $\sim 0.25 \mathrm{pc}$, from the SMBH. If the smaller black hole moves initially 

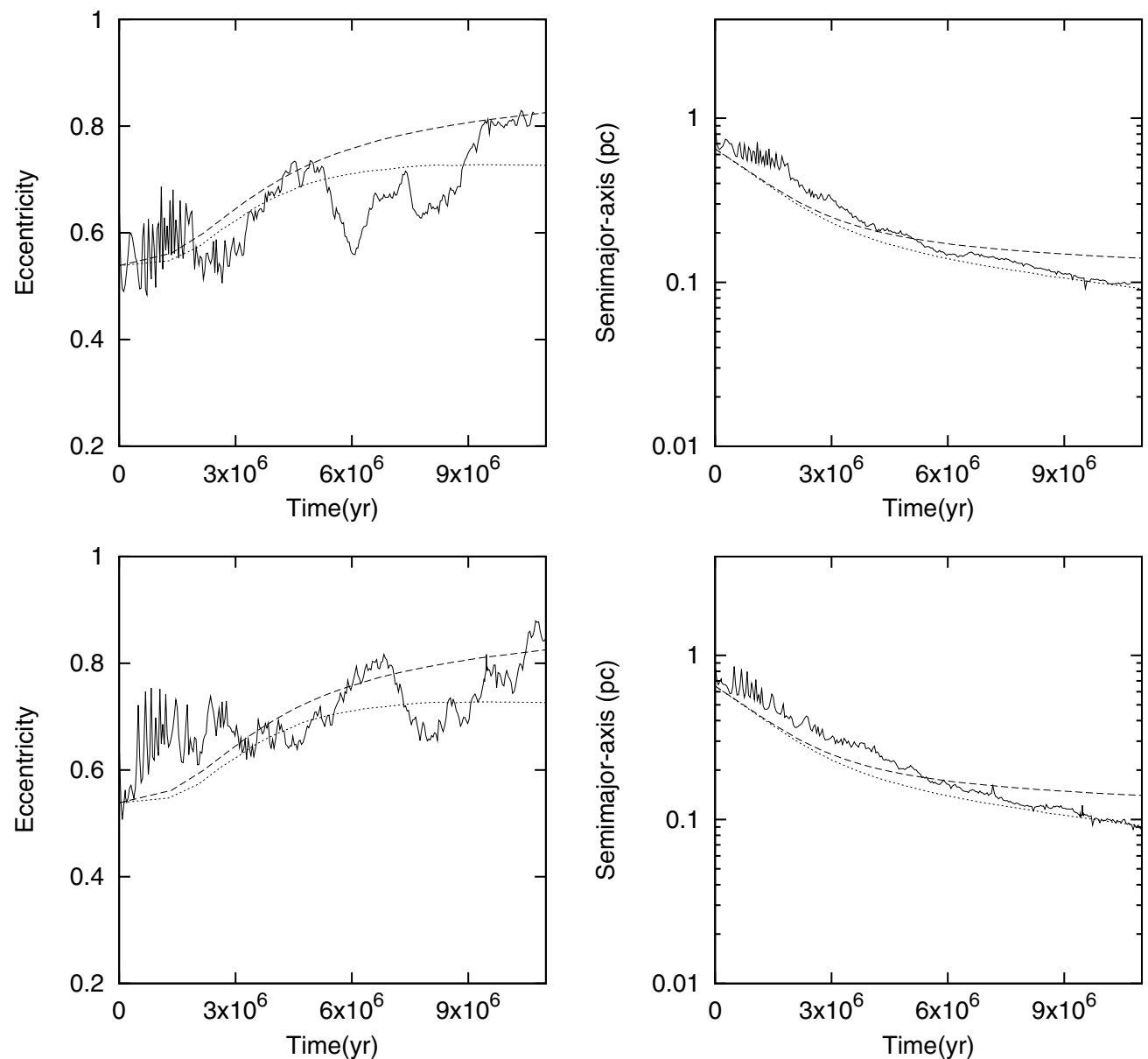

Figure 15. Evolution of eccentricity and semimajor axis for models G1 (upper panel) and G2 (lower panel) that differ only in the number of field particles: $N=200,000$ and 100,000 for models G1 and G2, respectively. Dashed lines are the theoretical predictions from Equation (18). Dotted lines were obtained with Equation (29) (i.e., including the frictional drag from stars with $v_{\star}>v$ ), where we used $p_{\max }=0.5 \mathrm{pc}$. As the black hole spirals in, its orbital eccentricity increases. This conclusion is quite robust, showing essentially no dependence on the number of background particles.
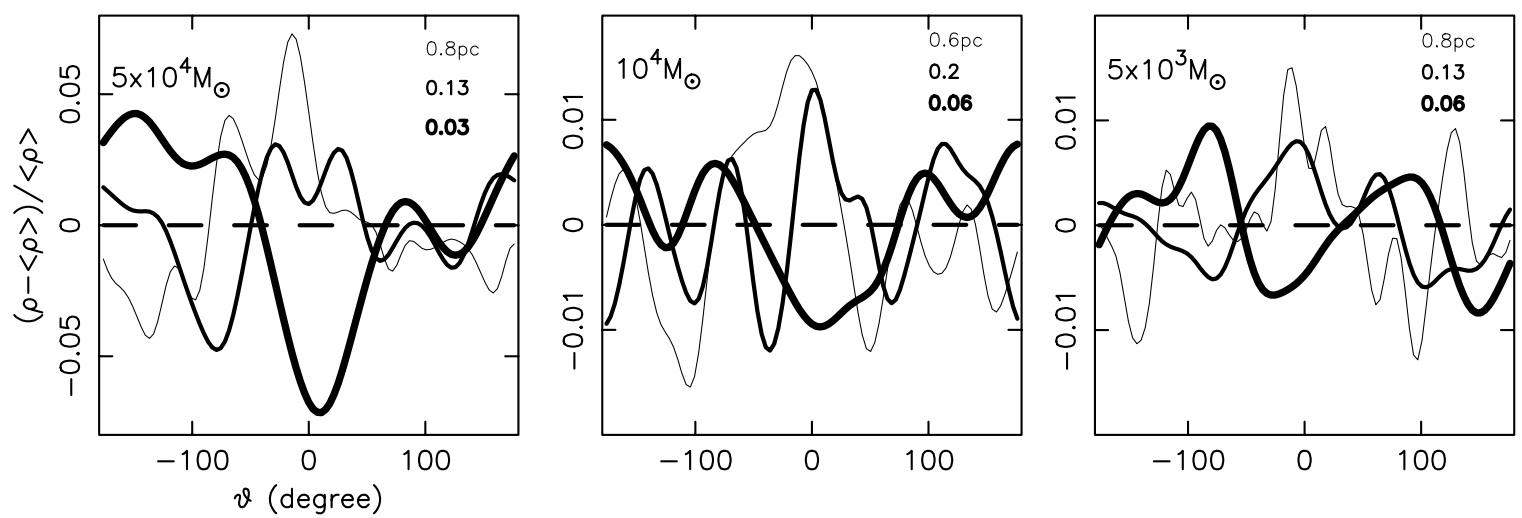

Figure 16. Relative overdensity in the $N$-body models for runs A1, E, and F along the black hole orbit. Line thickness decreases with increasing galactocentric distance. In the plots, the second black hole is always located at $\theta=0$ with $\dot{\theta}>0$.

on a non-circular orbit, its orbital eccentricity is predicted to increase with time due to the lower dynamical-friction force near periapsis.

Using $\mathrm{N}$-body simulations, we found that the frictional force never falls precisely to zero. As noted also by Chandrasekhar, stars moving faster than the test body contribute to the drag. When this contribution is included in the expression for the dynamical friction, Chandrasekhar's formula reproduces quite well the decay observed in $\mathrm{N}$-body simulations of the inspiral of a $\sim 1000 M_{\odot}$ black hole. The eccentricity increase predicted by
Chandrasekhar's theory is also confirmed. When the inspiralling object is more massive, a second mechanism contributes to the frictional force: the second black hole induces evolution of the background system, which tends to refill the initially empty regions of phase space.

In what follows, we discuss the implications of these results for a number of astrophysical problems related to the dynamics of massive bodies near the centers of galaxies. But first, we comment on how our $\mathrm{N}$-body results can be approximately scaled to systems with different masses and densities. 

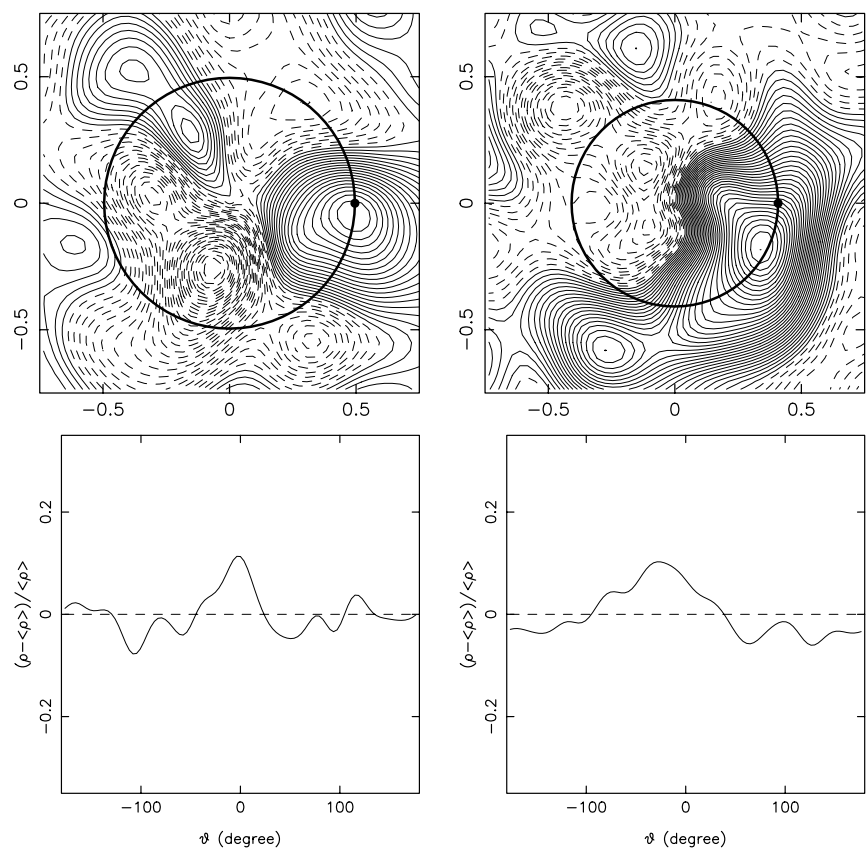
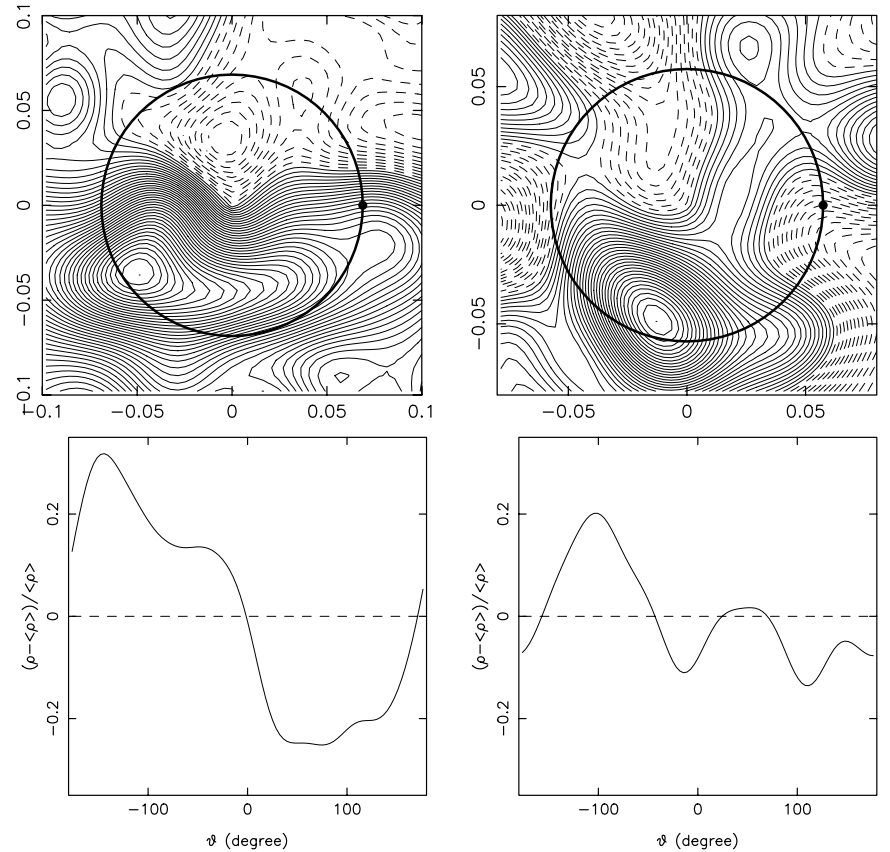

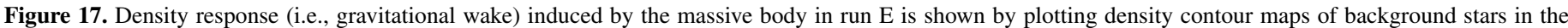

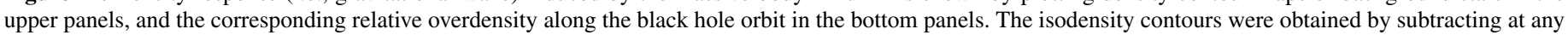

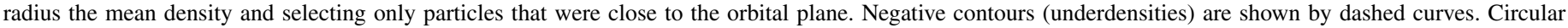
regions show the path over which the density was computed to obtain the plots in the bottom panels.

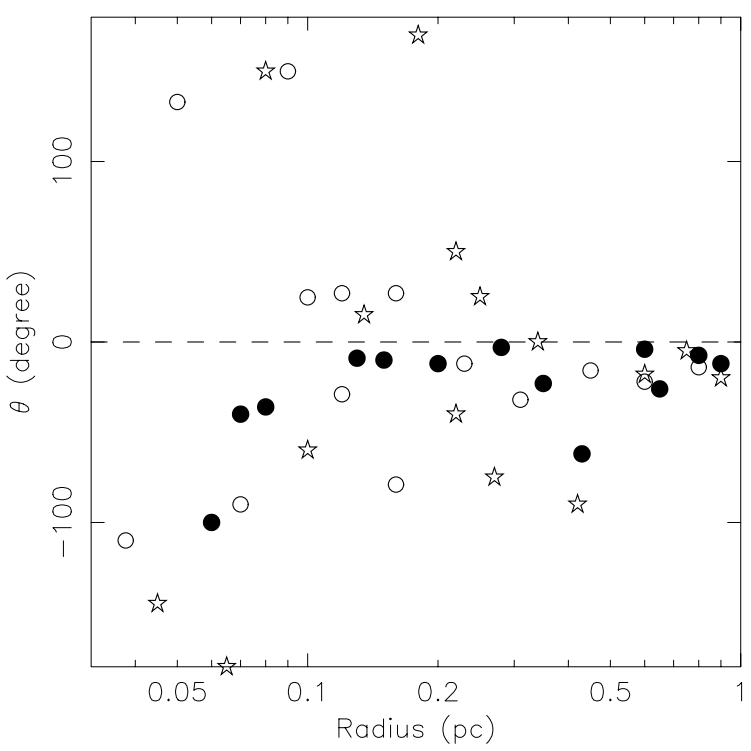

Figure 18. Position of the relative density maximum as a function of the black hole galactocentric radius in runs A1 (filled circles), E (open circles), and F (stars symbols). As in Figure 16, the $N$-body models were rotated such that the second black hole is located at $\theta=0$ with $\theta>0$.

The rate of inspiral of a massive body of mass $M$ is independent of the mass of field stars if $M \gg m$. Chandrasekhar's formula also predicts a linear dependence of the frictional force on $M,{ }^{1}$ and our simulations (as well as many others) confirm that prediction. If the density response of the background is ignored, the $N$-body results can then be scaled using

$$
r \rightarrow r \times\left[\frac{\tilde{r}\left(<3.4 \times 10^{-3} M_{\bullet}\right)}{0.1 \mathrm{pc}}\right]
$$

\footnotetext{
1 In its more general form (18), the dynamical-friction formula predicts an
} additional, approximately logarithmic dependence of force on $M$.

$$
\begin{aligned}
t \rightarrow t & \times\left[\frac{\tilde{r}\left(<3.4 \times 10^{-3} M_{\bullet}\right)}{0.1 \mathrm{pc}}\right]^{3 / 2}\left[\frac{M_{\bullet}}{4 \times 10^{6} M_{\odot}}\right]^{-1 / 2} \\
& \times\left[\frac{\tilde{M}}{M} \times \frac{4 \times 10^{6} M_{\odot}}{M_{\bullet}}\right]^{-1}
\end{aligned}
$$

where $\tilde{r}$ is radius containing a mass in stars $M_{\star}(<r) \approx$ $3.4 \times 10^{-3} M_{\bullet}, \tilde{M}$ is the mass of the test body, and $M$ its mass adopted in the $N$-body simulations of Table 1 . When in the simulations the background of stars evolves, the dependence on the mass of the infalling body becomes more complex; in this case, the appropriate scaling is obtained by setting $\tilde{M}=M \times\left[M_{\bullet} / 4 \times 10^{6} M_{\odot}\right]$, i.e., setting the ratio between the mass of the massive body and the central black hole the same as in the $N$-body simulation. Particular caution should also be taken when adopting $\tilde{M}>M$ since for large values of $\tilde{M}$ the massive body would perturb the background system more strongly than it does in $N$-body runs.

The condition that the background not evolve is satisfied in our simulations when $M \lesssim 10^{4} M_{\odot}$ and at early times in run E. We apply this approximate scaling to run A1, for which $M=5000 M_{\odot}$ and the total integration time is $\sim 1.5 \times 10^{7} \mathrm{yr}$. Assuming no change in the stellar density, replacing the massive body by a $\sim 10 M_{\odot}$ black hole increases the effective integration time by a factor of $\sim 500$, to $\sim 8 \times 10^{9} \mathrm{yr}$ (at which time the galactocentric radius is $\sim 0.06 \mathrm{pc}$ ). This result illustrates how - in the absence of a steep central density cusp-the time for stellar-mass black holes (BHs) to reach the center of the Galaxy from a starting radius of $\sim 1$ pc can easily exceed $\sim 10$ Gyr (a point we return to in Section 6.1).

Alternatively, we can identify our models with the center of a galaxy like M87, a luminous elliptical galaxy with a flat central density profile. We adopt $M_{\bullet}=3 \times 10^{9} M_{\odot}$ for the mass of the 
SMBH and we use a core velocity dispersion $\sigma_{v}=278 \mathrm{~km} \mathrm{~s}^{-1}$ and the relation $\sigma_{v}^{2}=4 \pi G \rho_{0}\left(r_{0} / 3\right)^{2}$ with $r_{0}=600 \mathrm{pc}$ to obtain the mass-density profile (Young et al. 1978; Lauer et al. 1995):

$$
\rho(r)=35 M_{\odot} \mathrm{pc}^{-3}\left(\frac{r}{600 \mathrm{pc}}\right)^{-\gamma}
$$

Taking $\gamma=0.6$, this gives a length normalization factor $\tilde{r} \approx 20 \mathrm{pc}$.

Runs $E$ and $F$. In these runs the background system evolves due to the perturbations induced by the massive body (see Figure 14). Setting $\tilde{M}=M \times\left[M_{\bullet} / 4 \times 10^{6} M_{\odot}\right]$, run E corresponds to the inspiral of a $\sim 7 \times 10^{6} M_{\odot}$ black hole starting from a distance of $200 \mathrm{pc}$, and a total integration time $\sim 2 \times 10^{9} \mathrm{yr}$. In the case of run $\mathrm{F}$, the inspiraling black hole would have a mass $\sim 4 \times 10^{7} \mathrm{M}_{\odot}$; it penetrates the inner $\sim 10 \mathrm{pc}$ in $\sim 3 \times 10^{8} \mathrm{yr}$ after which it effectively stalls.

Run A. The condition that the background not evolve is satisfied in runs A1, A2, and also at early times in run E. Setting $\tilde{M}=10^{6}\left(10^{5}\right) 10^{4} M_{\odot}$ in runs A1 and A2, the final integration time and orbital radius are $\sim 3 \times 10^{8}\left(10^{10}\right) 10^{12} \mathrm{yr}$ and $12 \mathrm{pc}$, respectively. This shows how, in the central core of a M87-like galaxy, the inspiral time for black holes of masses $\lesssim 10^{6} M_{\odot}$ could easily exceed a Hubble time (a point we further discuss in Section 6.2.2).

\subsection{Segregation of Massive Remnants at the Galactic Center}

About $1 \%$ of the total mass of the old population at the GC should be in the form of stellar-mass $\left(m \approx 10-20 M_{\odot}\right)$ BHs (Alexander 2005). Since stellar BHs are significantly more massive than the mean stellar mass $\left(\sim 1 M_{\odot}\right)$ expected for an evolved population, they would spiral into the center and segregate around the SMBH (Morris 1993). The time evolution of the remnant population depends sensitively on its initial distribution and also on the properties of the background distribution of lighter stars. In the case of a flat core in the stars, and a similar initial distribution in the $\mathrm{BHs}$, the time for the latter to reach a steady-state density profile can exceed a Hubble time, since the dynamical-friction force essentially ceases inside the core (Merritt 2010). On the other hand, if the stars follow a steep central density cusp, the mass density of BHs after $\sim 10$ Gyr can reach or exceed that of the other populations within $\sim 10^{-2} \mathrm{pc}$, leading to a quasi-steady-state density profile $n \simeq r^{-2}$ at small radii (e.g., Hopman \& Alexander 2006; Alexander \& Hopman 2009).

Understanding the distribution of BHs at the centers of galaxies like the Milky Way is crucial for making predictions about the expected event rate for low-frequency GW detectors (Hughes 2003). Since the stellar BHs at the GC are not directly detected, time-dependent inspiral calculations like the ones presented here provide the best hope of understanding their distribution. However, if the background stellar distribution is a flat core, our results show that a straightforward application of Chandrasekhar's formula can give misleading results.

Accordingly, we computed the evolution of a population of stellar BHs as they spiralled into the center of a galaxy with a flat stellar core, including the frictional force from the fast-moving stars. We began by generating random samples of positions and velocities from the isotropic distribution function corresponding to the density model of Equation (14) assuming $\gamma=0.6$; cores of various sizes, $r_{0}=(0.3,1,2) \mathrm{pc}$; and selecting only particles within $5 \mathrm{pc}$ of the SMBH. In each of these models, a total of 800 orbits (representing the stellar BHs) were then integrated by

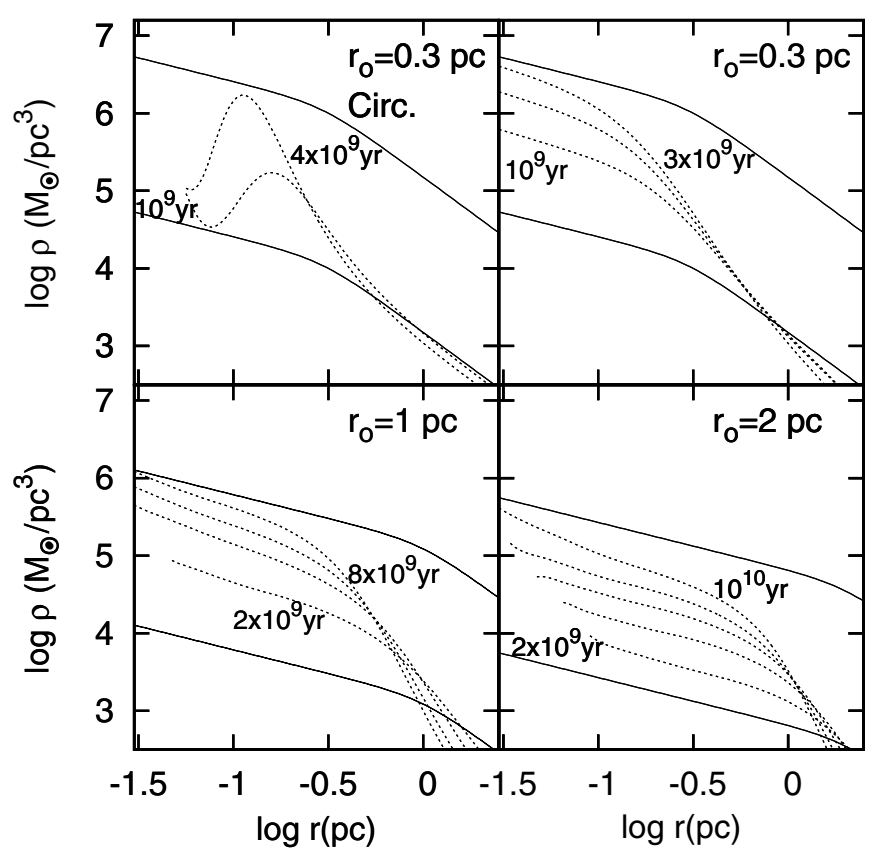

Figure 19. Evolution of the density of a population of $10 M_{\odot} \mathrm{BHs}$ (dotted curves) assuming that they constitute $1 \%$ of the total mass density initially. Results are displayed for three choices of the core parameter $r_{0}=(0.3,1,2) \mathrm{pc}$. Lower (upper) solid lines show the initial density profile of stellar BHs (stars). In the upper left panel, the BHs lie on circular orbits, while in the other cases we assume an isotropic initial distribution of velocities. Density profiles are shown at time intervals of $\Delta t=2 \times 10^{9} \mathrm{Gyr}$ in the lower panels, while $\Delta t=10^{9} \mathrm{Gyr}$ in the upper right panel.

solving the system of Equations (20), with dynamical-friction force given by

$$
\begin{aligned}
\boldsymbol{f}_{\mathrm{fr}}= & -4 \pi G^{2} M \rho(r) \frac{\boldsymbol{v}}{v^{3}}(F(<v, r) \ln \Lambda \\
& \left.+\int_{v}^{\sqrt{-2 \phi(r)}} d v_{\star} 4 \pi f\left(v_{\star}\right) v_{\star}^{2}\left[\ln \left(\frac{v_{\star}+v}{v_{\star}-v}\right)-2 \frac{v}{v_{\star}}\right]\right),
\end{aligned}
$$

with $\ln \Lambda=15, M=10 M_{\odot}$. At each time, the density profile and eccentricity distribution of the inspiralling objects were computed by sampling each orbit over time intervals of $0.3 \mathrm{Gyr}$. We also considered one model with core parameter $r_{0}=0.3 \mathrm{pc}$ in which all BHs were initially on circular orbits.

All of the calculations presented in this section assume that the mass density due to the BHs remains small compared with the mass density in stars and that the stellar distribution is unchanging. Because the two-body relaxation time for $1 M_{\odot}$ stars is so long in these models, and $\sim 10$ times longer than the black hole inspiral time, ignoring the evolution of the stellar distribution due to star-star encounters is reasonable. This basic assumption is also supported by recently published $N$ body simulations (Gualandris \& Merritt 2011) that show how, in models with a pre-existing stellar core, the distribution of BHs evolves against an essentially fixed background of stars. However, once the density in BHs begins to approach that in the stars, our calculations are no longer valid.

In Figure 19 we plot the density profile of $\mathrm{BHs}$ at different times, assuming that their fraction is initially $10^{-2}$ of the total mass density. The upper panels give the results for the model with $r_{0}=0.3 \mathrm{pc}$. In these integrations, the core is very small and after only $\sim 1$ Gyr the density of black hole rises very steeply 


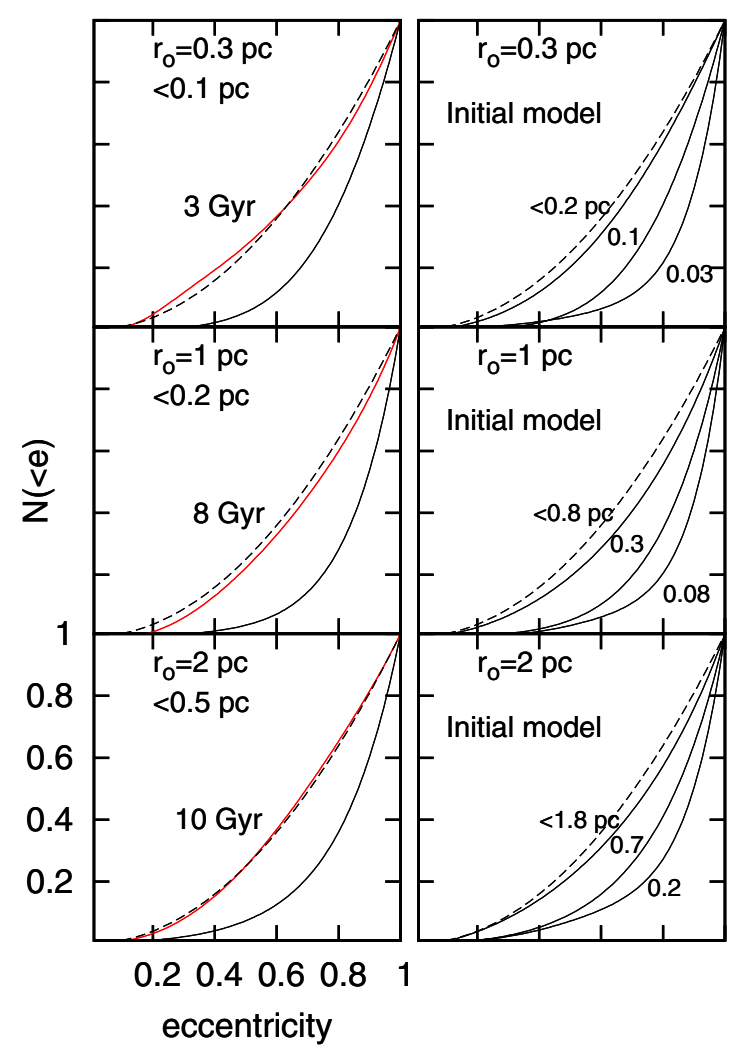

Figure 20. Left panels: final cumulative eccentricity distribution of stellar BHs for the integrations displayed in Figure 19 (red curves) that would be measured inside the core within some radius. Solid curves give the initial distributions. Right panels: cumulative eccentricity distributions of the initial models (solid curves) evaluated within different radii. At small galactocentric radii, the distribution is dominated by high-eccentricity orbits, in spite of the fact that the velocity distribution is isotropic. Dashed curves show for comparison a "thermal" eccentricity distribution, $N \sim e^{2}$.

(A color version of this figure is available in the online journal.)

going into the stellar core. After $\sim 4$ Gyr the BHs accumulate at radii near the core, matching the density in stars at $\sim 0.01 \mathrm{pc}$. In the circular-orbit model, the density profile at $1 \mathrm{Gyr}$ shows a maximum at $\sim 0.2 \mathrm{pc}$ that grows and migrate inward due to the friction produced by fast-moving stars inside these radii. The evolution for the isotropic run is comparably rapid and after $\sim 3$ Gyr the density of BHs reaches that in stars at $\sim 0.01 \mathrm{pc}$.

Merritt (2010) showed that a core of the size currently observed is a natural consequence of two-body relaxation acting over $10 \mathrm{Gyr}$, starting from a core of radius $\sim 1 \mathrm{pc}$. It is therefore of interest to study the evolution of the black hole distribution in models with parsec-scale cores. This is shown in the lower panels of Figure 19. In these cases the evolution is slower as a consequence of the increased size of the stellar core, and even after 10 Gyr the density of BHs can remain substantially lower than that in stars at all radii. We conclude that it would be unjustified to assume that the massive remnants have yet reached a steady-state density profile at the GC. One consequence is that rates of capture of stellar BHs by the SMBH at the GC (EMRIs) may be much lower than in standard models that postulate a collisionally relaxed nucleus (e.g., Hopman \& Alexander 2006).

The left panels of Figure 20 plot the cumulative distribution of eccentricities of $\mathrm{BHs}$ inside various radii. Since the final eccentricity of each orbit is larger than its initial value (see Section 2.2), one might naively expect the eccentricity distributions to evolve toward a form that is increasingly strongly peaked near $e \approx 1$. This would be the case if one plotted $N(e)$ for a fixed subset of objects. However, when restricting the sample to a given radial range, the result is very different. The reason (e.g., Merritt 2010, Appendix) is illustrated in the righthand panels of Figure 20: given a flat density profile, even an isotropic distribution of objects around a SMBH will have an eccentricity distribution that is strongly peaked near $e=1$, since the only objects that can approach closely to the SMBH are on highly eccentric orbits. As the distribution of BHs evolves away from this initial configuration, the regions of low-energy phase space that were initially empty are gradually refilled, and the eccentricity distribution begins to approach more closely to a "thermal" form, $N(<e) \propto e^{2}$. In addition, (1) the eccentricity of individual orbits inside the core grows only very slowly since they are in a region where the dynamical-friction force is small (see Figure 3); (2) the eccentricity of BHs initially beyond the core decreases initially since they lie in a $\gamma \approx 1.8$ cusp; their eccentricities subsequently increase as the orbital periapsis enters the core, but in most cases this second phase is too short (see Figure 4) to produce final eccentricities significantly different from the initial values. We finally computed the anisotropy parameter (32) at the final integration time, defined as the time when the mass density in BHs reaches that in stars at small radii, and found that the departures from isotropy remained small at all radii.

\subsection{Dynamical Evolution of Eccentric Black Hole Binaries}

Gravitational radiation emitted by binary black holes with masses $10^{3}-10^{7} M_{\odot}$ is the principal target of planned, spacebased, GW observatories. In the present literature the strain amplitude of the GW radiation is typically obtained under the assumption of complete circularization of the binary at the moment that the signal enters into the observable band. This simplification is motivated by the predicted strong eccentricity decay when binary dynamics are dominated by relativistic effects. The expressions of the time average change of eccentricity $e$ and semimajor axis $a$ in the relativistic regime of a binary with components of masses $m_{1}$ and $m_{2}$ were derived by Peters \& Matthews (1963):

$$
\begin{gathered}
\left\langle\frac{d a}{d t}\right\rangle=-\frac{64}{5} \frac{G^{3} m_{1} m_{2}\left(m_{1}+m_{2}\right)}{c^{5} a^{3}} f(e), \\
\left\langle\frac{d e}{d t}\right\rangle=-\frac{304}{15} \frac{G^{3} m_{1} m_{2}\left(m_{1}+m_{2}\right)}{c^{5} a^{4}\left(1-e^{2}\right)^{5 / 2}}\left(e+\frac{121}{304} e^{3}\right),
\end{gathered}
$$

where $c$ is the speed of light and

$$
f(e)=\left(1-e^{2}\right)^{-7 / 2}\left(1+\frac{73}{24} e^{2}+\frac{37}{96} e^{4}\right) .
$$

The strong dependence of the enhancement factor $f(e)$ on $e$ shows the fundamental role of the binary eccentricity in determining the rate at which the system loses energy due to GW emission.

A way to follow the orbital inspiral of a massive body at the $\mathrm{GC}$, due both to dynamical-friction and $\mathrm{GW}$ radiation, is to couple Chandrasekhar's formula for the frictional drag with the 2.5 post-Newtonian equations representing GW energy loss (Merritt 2012). In the limit $M / M_{\bullet} \ll 1$, the total deceleration 

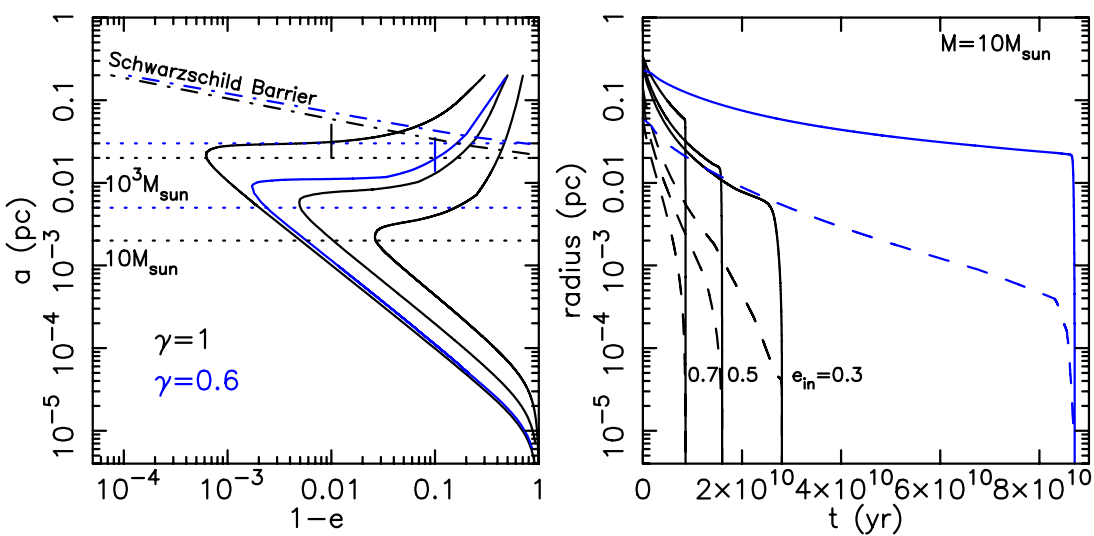

Figure 21. Left panel: evolutionary tracks of a massive object in the Galactic center starting from various eccentricities $e_{\text {in }}=(0.3,0.5,0.7)$, from an initial semimajor axis $a_{\text {in }}=0.2 \mathrm{pc}$ and adopting two different inner slopes of the mass-density profile $\gamma=(1,0.6)$. Dot-dashed lines are the Schwarzschild barrier, Equation (45), below which resonant relaxation is suppressed by relativistic precession and gravitational scattering is dominated by classical non-resonant relaxation. Vertical marks give the radii within which the two-body relaxation timescale for changes in angular momentum $\left(t_{r, \text { eff }}\right)$ becomes shorter than the timescale of evolution for angular momentum in our integrations $\left(t_{\mathrm{evol}}\right)$, assuming $10 M_{\odot}$ for the mass of the inspiraling black hole. Inside these radii, for $M \leqslant 10 M_{\odot}$, our integrations are no longer valid since two-body scattering, rather than dynamical friction, would dominate the orbital evolution. For $e_{\text {in }} \lesssim 0.5$ and $\gamma=1$ (two rightmost curves), at any radius, $t_{r \text {,eff }}$ was always longer than $t_{\mathrm{evol}}$ and no vertical marks are displayed. In the two leftmost curves, the condition that $t_{r \text {,eff }}>t_{\text {evol }}$ at any radius would instead require a slightly larger mass for the $\mathrm{BH}: M \gtrsim 15 M_{\odot}$. This shows that gravitational scattering from stars can be neglected and our integrations are valid for relatively small masses of the test particle. Within the Schwarzschild barrier, dynamical friction is therefore the main mechanism inducing creation of EMRIs. We also stress that in these integrations, changes in the stellar distribution are not taken into account. For instance, the stellar potential would be strongly perturbed when the mass of the inspiraling black hole becomes comparable to the mass in stars contained inside its orbital radius. As a reference, dotted lines in the panel display the radius within which the mass in stars in the model is 10 or $1000 M_{\odot}$. Right panel: time evolution of periapsis (dashed lines) and apoapsis (continue line) for a $10 M_{\odot} \mathrm{BH}$. The sinking timescale decreases with increasing the initial eccentricity, and, for the set of computed orbits, it is shorter than $10^{10}$ yr only for $e_{\text {in }}=0.7$ (leftmost curve in the panel).

can be approximated by

$$
\begin{aligned}
\boldsymbol{f}= & -4 \pi G^{2} M \rho(r) \frac{\boldsymbol{v}}{v^{3}}(F(<v, r) \ln \Lambda \\
& \left.+\int_{v}^{\sqrt{-2 \phi(r)}} d v_{\star} 4 \pi f\left(v_{\star}\right) v_{\star}^{2}\left[\ln \left(\frac{v_{\star}+v}{v_{\star}-v}\right)-2 \frac{v}{v_{\star}}\right]\right) \\
& -G M[A \boldsymbol{n}+B \boldsymbol{v}],
\end{aligned}
$$

where $\boldsymbol{n}=\boldsymbol{r} / \boldsymbol{r}$ and

$$
\begin{gathered}
A=\frac{1}{c^{5}}\left[-\frac{24 v_{r} v^{2} G M_{\bullet}}{5 r^{3}}-\frac{136 v_{r} G^{2} M_{\bullet}^{2}}{15 r^{4}}\right] ; \\
B=\frac{1}{c^{5}}\left[\frac{8 v^{2} G M_{\bullet}}{5 r^{3}}+\frac{24 G^{2} M_{\bullet}^{2}}{5 r^{4}}\right],
\end{gathered}
$$

with $v_{r}$ the radial component of the velocity vector. Evidently, both the frictional force and the 2.5PN correction are dissipative terms, but, while the latter term always drives to lower eccentricities, the effect of dynamical friction on the orbital eccentricity has a strong dependence on the phase-space distribution associated with the stellar background (see Section 3.2). Note that, in Equation (40), if we neglect the dependence of the Coulomb logarithm on the mass of the test body, both the frictional term and the post-Newtonian terms depend linearly on $M$ implying that the time evolution of the orbital elements can be trivially rescaled to any $M$ as long as the condition $M / M_{\bullet} \ll 1$ holds (see also Equations (36) and (37)).

\subsubsection{Dynamical Friction in the Context of the EMRI Problem}

Extreme-mass-ratio inspirals (EMRIs) are a potential source of low-frequency GWs (Hughes 2003; Barack \& Cutler 2004; Amaro-Seoane et al. 2007). In steady-state models of the GC, the distributed mass within $10^{-2} \mathrm{pc}$ of the SMBH is dominated by stellar BHs (Hopman \& Alexander 2006). At these radii, dynamical friction is therefore typically ignored and it is assumed that captures for EMRIs are driven by gravitational scattering from other stellar BHs (e.g., Merritt et al. 2011). On the other hand, if the background stellar distribution has a flat core, the density of BHs can remain small compared with the mass density of other populations (e.g., Gualandris \& Merritt 2011; Section 6.1). Under these circumstances, at any radius, massive remnants might see a background whose density comes mostly from lighter stars and dynamical friction becomes a competing mechanism in driving capture of EMRIs.

Using Equation (40), we computed the trajectory of the test mass under a variety of assumptions for the background system. Results of these integrations are displayed in Figure 21. We considered orbits of different initial eccentricities $e_{\text {in }}=$ $(0.3,0.5,0.7)$, starting from a semimajor axis $a_{\text {in }}=0.2 \mathrm{pc}$. For the stellar background we used the density model of Equation (14) with two different values of the internal slope index: $\gamma=1$ (black lines) and 0.6 (blue lines). For an eccentric orbit in a flattened cusp, dynamical friction at apoapsis dominates the evolution causing a rapid increase of the orbital eccentricity. In the simplified picture in which the frictional force at periapsis is vanishing small, the apoapsis distance remains unchanged in time, while the periapsis becomes progressively smaller; at some point, the minimum distance from the SMBH is small enough that the $2.5 \mathrm{PN}$ terms start to dominate the evolution. The drag at periapsis then circularizes the orbit and causes the merger of the two black holes.

Near a SMBH, as long as the relativistic precession timescale is much longer than the orbital period, the mechanism that dominates the scattering of stars onto high-eccentricity orbits is resonant relaxation. Because in the potential of a point mass the orbits are fixed ellipses, perturbations on a test particle are not random but correlated (Rauch \& Tremaine 1996). The residual torque $|\boldsymbol{T}| \approx \sqrt{N} G m / r$, exerted by the $N$ randomly 
oriented, orbit-averaged mass distributions of the surrounding stars, induces coherent changes in angular momentum $\Delta \boldsymbol{L}=\boldsymbol{T} t$ on times $t \lesssim t_{\text {coh }}$, where the coherence time $t_{\text {coh }}$ is fixed by the mechanism that most rapidly causes the orbits to precess (e.g., mass precession, relativistic precession). The angular momentum relaxation time associated with resonant relaxation is

$$
t_{r r}=\left(\frac{L_{c}}{\Delta L_{\mathrm{coh}}}\right)^{2} t_{\mathrm{coh}}
$$

where $L_{c} \equiv \sqrt{G M_{\bullet} a}$ is the angular momentum of a circular orbit and $\left|\Delta L_{\text {coh }}\right| \sim\left|\boldsymbol{T} t_{\text {coh }}\right|$ is the accumulated change over the coherence time. Assuming that the precession is determined by the mean field of stars, the angular momentum relaxation time becomes (Rauch \& Tremaine 1996)

$$
t_{r r} \approx 2.9 \times 10^{7} \mathrm{yr}\left(\frac{M_{\bullet}}{4 \times 10^{6} M_{\odot}}\right)^{1 / 2}\left(\frac{a}{0.1 \mathrm{pc}}\right)^{3 / 2}\left(\frac{m}{M_{\odot}}\right)^{-1} .
$$

Dot-dashed lines in the left panel of Figure 21 give the Schwarzschild barrier. Above these lines, resonant relaxation is the most rapid mechanism affecting angular momenta; below the curves, relativistic precession becomes efficient at suppressing resonant relaxation and the gravitational perturbations are dominated by classical "two-body" relaxation. The value of the angular momentum that defines the Schwarzschild barrier is (Merritt et al. 2011)

$$
\begin{aligned}
\left(1-e^{2}\right)_{\mathrm{SB}} \approx & 5.8 \times 10^{-3}\left(\frac{C_{\mathrm{SB}}}{0.7}\right)^{2}\left(\frac{a}{0.1 \mathrm{pc}}\right)^{-2}\left(\frac{M_{\bullet}}{4 \times 10^{6} M_{\odot}}\right)^{4} \\
& \times\left(\frac{m}{M_{\odot}}\right)^{-2}\left(\frac{N}{10^{4}}\right)^{-1},
\end{aligned}
$$

where $N$ is the number of stars within radius $a$ and $C_{\mathrm{SB}}$ is a constant of order of unity. Beyond the barrier, the time for encounters to change the orbital angular momentum by of order itself is $t_{r \text {,eff }}=2(1-e) t_{\mathrm{r}}$, where for the non-resonant relaxation timescale we adopt the approximate expression (Hopman \& Alexander 2006)

$$
\begin{aligned}
t_{r} \approx & 4.8 \times 10^{10} \mathrm{yr}\left(\frac{a}{0.1 \mathrm{pc}}\right)^{3 / 2}\left(\frac{M_{\bullet}}{4 \times 10^{6} M_{\odot}}\right)^{3 / 2} \\
& \times\left(\frac{m}{M_{\odot}}\right)^{-2}\left(\frac{N}{10^{4}}\right)^{-1} .
\end{aligned}
$$

For our integrations to be viable, the timescale for dynamical friction to change the orbital angular momentum, $t_{\mathrm{evol}} \sim$ $(1-e)|\mathrm{d}(1-e) / \mathrm{d} t|^{-1}$, must be shorter than $t_{r, \text { eff }}$ at all radii. Vertical marks in the left panel of Figure 21 give the orbital radius within which $t_{r \text {,eff }}$ becomes smaller than $t_{\text {evol }}$ assuming $M=10 M_{\odot}$. For $e_{\text {in }}=(0.3,0.5)$ and $\gamma=1$ (two rightmost curves), at any radius, $t_{r \text {,eff }}$ was always larger than $t_{\mathrm{evol}}$ and no vertical marks are displayed. Increasing the initial eccentricity to $e_{\text {in }}=0.7, t_{r \text {,eff }}$ equals $t_{\text {evol }}$ at $\sim 0.03 \mathrm{pc}$. At smaller radii, two-body relaxation would dominate the orbital evolution and, for a $10 M_{\odot}$ black hole, this integration is not longer valid. Taking $e_{\text {in }}=0.5$ and $\gamma=0.6$ (blue curve in the figure) this transition occurs at $\sim 0.02 \mathrm{pc}$. Because increasing the mass of the test body reduces $t_{\mathrm{evol}}$ but leaves $t_{r, \text { eff }}$ unchanged, it is always possible to set $M$ such that the condition $t_{r \text {,eff }}>t_{\text {evol }}$ is satisfied everywhere within the Schwarzschild barrier. In these two latter cases this condition requires a slightly larger mass of the test body: $M \gtrsim 15 M_{\odot}$ (Woosley et al. 2002). Two-body scattering effects from field stars can therefore be ignored for relatively small masses of the sinking black hole. We conclude that, in a flat density distribution near a SMBH and at radii relevant for the EMRI problem $(<0.01 \mathrm{pc})$, dynamical friction might be an important process in driving the formation of EMRIs.

Gravitational scattering can be dominated by other stellar BHs if their density becomes comparable of that in stars at small radii as a consequence of mass segregation. In an unsegregated model, the number of stellar BHs (of mass $10 M_{\odot}$ ) is predicted to be $10^{-3}$ times that in stars. From Equations (45) and (46) it follows that, in this case, the scattering from black holes can be ignored with respect to the perturbations from the stellar population. Gravitational scattering from black holes starts to compete with that from stars when their number at small radii $(\sim 1 \mathrm{mpc})$ is $10^{-2} \times N$, similar to that found at later times in Figure 19 for $r_{0} \sim 2$ pc. In relaxed mass-segregated models, instead, the number of black holes would be approximately $N$, and they will dominate the orbital evolution of the test mass at any radius inside the Schwarzschild barrier (Alexander \& Hopman 2009).

Finally, we note that dynamical friction can be very inefficient if the mass of the inspiraling object becomes comparable to the mass in stars within its orbital radius. In the $\gamma=1$ cusp for a $\sim 1000(10) M_{\odot}$, this occurs at $\sim 0.02(0.002)$ pc or at $\sim 0.03(0.005)$ pc when $\gamma=0.6$. This suggests that the results of Figure 21 may not apply for large masses of the test body and for small initial eccentricities $(\lesssim 0.3)$. Accurate $N$-body simulations, including high-order post-Newtonian terms, should be used to better understand at which extends the conclusions made here can be applied. We reserve this study to a future paper.

In order for an extra-galactic source to be observable by proposed space-based interferometers, it must have an orbital frequency $\gtrsim 10^{-4} \mathrm{~Hz}$ (Amaro-Seoane et al. 2007) or

$$
a \lesssim a_{f} \equiv 4 \times 10^{-3} \operatorname{mpc}\left(\frac{M_{\bullet}}{4 \times 10^{6} M_{\odot}}\right)^{1 / 3}
$$

We explored whether the computed orbits would retain some degree of eccentricity by the time the binary enters the instrumental sensitivity window, by evaluating the eccentricity, $e_{f}$, at the time at which the condition (47) is satisfied and comparing this value with the minimum eccentricity that would require non-circular templates for data analysis: $e \sim 10^{-4}$ (Porter \& Sesana 2010). We note that strong sources (with high eccentricity) might be detectable at lower frequencies (i.e., larger semimajor axis; Amaro-Seoane et al. 2007). The use of Equation (47) is therefore a conservative one.

Figure 22 plots $e_{f}$ as a function of the initial eccentricity for the orbits displayed in Figure 21. In addition, we computed a set of orbits with different initial eccentricities by removing from Equation (40) the dynamical-friction term. Each dotdashed curve in the figure corresponds to a fixed value for the coalescence time (Peters 1964):

$$
\begin{aligned}
t_{m} \simeq & 3.6 \times 10^{12} \mathrm{yr}\left(\frac{10 M_{\odot}}{M}\right)\left(\frac{4 \times 10^{6} M_{\odot}}{M_{\bullet}}\right)^{2} \\
& \times\left(\frac{a}{\mathrm{mpc}}\right)^{4}\left(1-e^{2}\right)^{7 / 2}
\end{aligned}
$$

Taking $M=10 M_{\odot}\left(1000 M_{\odot}\right)$, this corresponds to $t_{m}=10^{15}$, $10^{14}, \cdots, 10^{8} \mathrm{yr}\left(10^{13}, 10^{12}, \cdots, 10^{6} \mathrm{yr}\right)$ from the bottom to the 


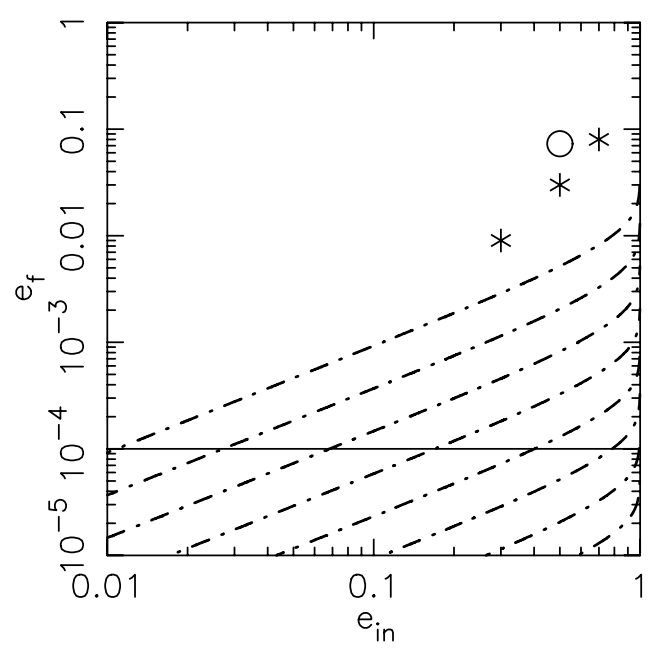

Figure 22. Eccentricity at the moment the binary enters the sensitivity window of planned space-based interferometers, $e_{f}$, as a function of the initial orbital eccentricity $e_{i n}$ for the integrations displayed in Figure 21. Star symbols are for $\gamma=1$ (black curves in Figure 21), empty circle for $\gamma=0.6$ (blue curve in Figure 21). The dot-dashed lines give $e_{f}$ ignoring dynamical friction. For a given initial eccentricity and secondary black hole mass, we fixed the merger time by using Equation (48) and varying the initial orbital semimajor axis. If we take a test mass of $10 M_{\odot}\left(1000 M_{\odot}\right)$ this corresponds to merger times of $10^{15}, 10^{14}, \cdots, 10^{8} \mathrm{yr}\left(10^{13}, 10^{12}, \cdots, 10^{6} \mathrm{yr}\right)$ from bottom to top line. As comparison, the orbital eccentricity and merger time for the integrations of Figure 21, at the moment GW energy loss stars to dominate the evolution, are (from left to right of that figure) $e \sim(0.9994,0.998,0.994,0.97)$ and $t_{m} \sim\left(5.9 \times 10^{7}, 1 \times 10^{8}, 1.9 \times 10^{9}, 5 \times 10^{9}\right)$ yr. Horizontal line represents approximately the lowest value of $e_{f}$ that would require non circular templates for data analysis ( $e \sim 10^{-4}$; Porter \& Sesana 2010).

top line, respectively. It is evident that even for relatively low initial eccentricities and large merger times the binary will have a value of $e_{f}$ significantly different from zero. This study suggests that secondary black holes typically reach the GW radiation regime on wide orbits that are still very eccentric, and even after the semimajor axis has decreased to values small enough for detection by space-based interferometers, eccentricities can be large enough that the efficient analysis of GW signals would require the use of eccentric templates (see also Barack \& Cutler 2004).

\subsubsection{Orbital Decay in the Cores of Giant Elliptical Galaxies}

Until the discovery of a stellar core in the Milky Way (Buchholz et al. 2009; Do et al. 2009; Bartko et al. 2010), the density was generally assumed to follow a steep power law, $\rho \sim r^{-2}$, inside the influence radius of Sgr A*. The same assumption is still commonly made when modeling the so-called power-law galaxies: galaxies of low to moderate luminosity that also exhibit steeply rising densities near the center (Gebhardt et al. 1996; Faber et al. 1997). Whether other power-law galaxies will turn out to harbor parsec-scale cores like the one in the Milky Way remains to be seen. But it has long been known that cores are ubiquitous in stellar spheroids brighter than $\sim 10^{10} L_{\odot}$, whose influence radii can be resolved (Ferrarese et al. 1994; Lauer et al. 1992). Core sizes are observed to be of order the $\mathrm{SMBH}$ influence radius or somewhat greater, consistent with models in which the cores are produced by the scouring effect of binary SMBHs (Merritt 2006; Gualandris \& Merritt 2011).

In this section, we use Equation (40) to investigate the orbital evolution of a massive black hole that spirals into the center of a giant elliptical galaxy with a core. We base our models on M87. The relevant properties of M87 are summarized at the start of this section. Here we note that the core of M87 extends substantially beyond the $\mathrm{SMBH}$ influence radius: $r_{c} / r_{\mathrm{bh}} \approx 600 \mathrm{pc} / 200 \mathrm{pc} \approx 3$. By comparison, the Milky Way has $r_{c} \approx 0.3 r_{\mathrm{bh}}$. This difference may reflect different formation processes for the two cores or may be a result of the shorter relaxation time at the center of the Milky Way, which could cause the core to shrink over 10 Gyr (Merritt 2010).

Following the evolution of a binary SMBH at the center of a galaxy requires self-consistent simulations that can correctly treat the response of the background stars to the presence of the second massive body (e.g., Ebisuzaki et al. 1991; Quinlan \& Hernquist 1997; Milosavljević \& Merritt 2001). Here, we limit ourselves to the case where the inspiralling black hole is much less massive than the central one. For instance, capture of a Milky Way sized galaxy by M87 would bring a second SMBH into the center forming a binary of mass ratio $\sim 10^{-3}$. This problem can be seen as a scaled-down version of the capture of an intermediate-mass black hole by Sgr A*. Simulations of the latter scenario (e.g., Baumgardt et al. 2006) have generally assumed a steeply rising stellar density around the SMBH; inspiral of the intermediate-mass black hole is found to stall when the semimajor axis of the binary drops to $\sim 10^{-3} \mathrm{pc}$, the radius at which the binary is able to eject stars with greater than escape velocity. When there is a pre-existing core, the binary evolves somewhat differently than in these simulations; as we showed above, the orbital periapsis progressively decreases while the apoapsis hardly changes. As a result, the orbital semimajor axis can still be large at the time that GW losses becomes significant. Since most of the frictional force occurs near apoapsis, we do not expect significant stalling or core depletion to occur until late in the evolution, perhaps not before the two black holes merge (e.g., Fukushige et al. 1992). Nevertheless, in what follows, we will explicitly note when in our integrations the mass of the sinking object becomes comparable to the mass in stars enclosed within its orbital radius.

We carried out calculations using the mass-density profile of Equation (14) with $\left\{\alpha=1 ; \gamma_{e}=1.8 ; \gamma=0.5 ; r_{0}=\right.$ $\left.600 \mathrm{pc} ; \rho_{0}=35 M_{\odot} \mathrm{pc}^{-3}\right\}$ and $M_{\bullet}=3 \times 10^{9} M_{\odot}$. The left panel of Figure 23 gives the orbital evolution of a test particle starting from an orbital radius of $100 \mathrm{pc}$ and eccentricity $e_{\text {in }}=(0.5,0.7,0.9)$. Dotted lines in the panel represent the radii at which the stellar mass enclosed in the orbit is $10^{3}$ or $4 \times 10^{6} M_{\odot}$. For the two more eccentric orbits (two leftmost curves), it is possible that the binary enters the GW regime before violating these conditions.

Although in our model the binary black hole mass is above the range $\left(10^{3}-10^{7} M_{\odot}\right)$ normally associated with space-based interferometers, we can nevertheless ask whether the eccentricity would remain large after the massive binary has entered into the GW regime. The Schwarzschild radius of a $3 \times 10^{9} M_{\odot}$ $\mathrm{SMBH}$ is $r_{\mathrm{SC}} \approx 1.4 \times 10^{-4} \mathrm{pc}$. When the orbital semimajor axis is $10 \times r_{\mathrm{SC}}$, we find that the binary eccentricity is still very large: $e \approx(0.08,0.6,0.8)$ for $e_{\text {in }}=(0.5,0.7,0.9)$. When $a=5 \times r_{\mathrm{SC}}$ the corresponding eccentricity is $e \approx(0.03,0.4,0.7)$.

The blue curves in Figure 23 were obtained by computing the frictional drag using Chandrasekhar's formula in its most common form, which assumes a locally Maxwellian distribution of velocities (Equation (31)). This approximation results in a very different orbital evolution characterized by smaller orbital eccentricities (for a given semimajor axis) and faster orbital decay when compared with the results obtained using the more correct formula (40). We note that-in spite of a higher rate of orbital decay - the smaller eccentricities achieved during the 

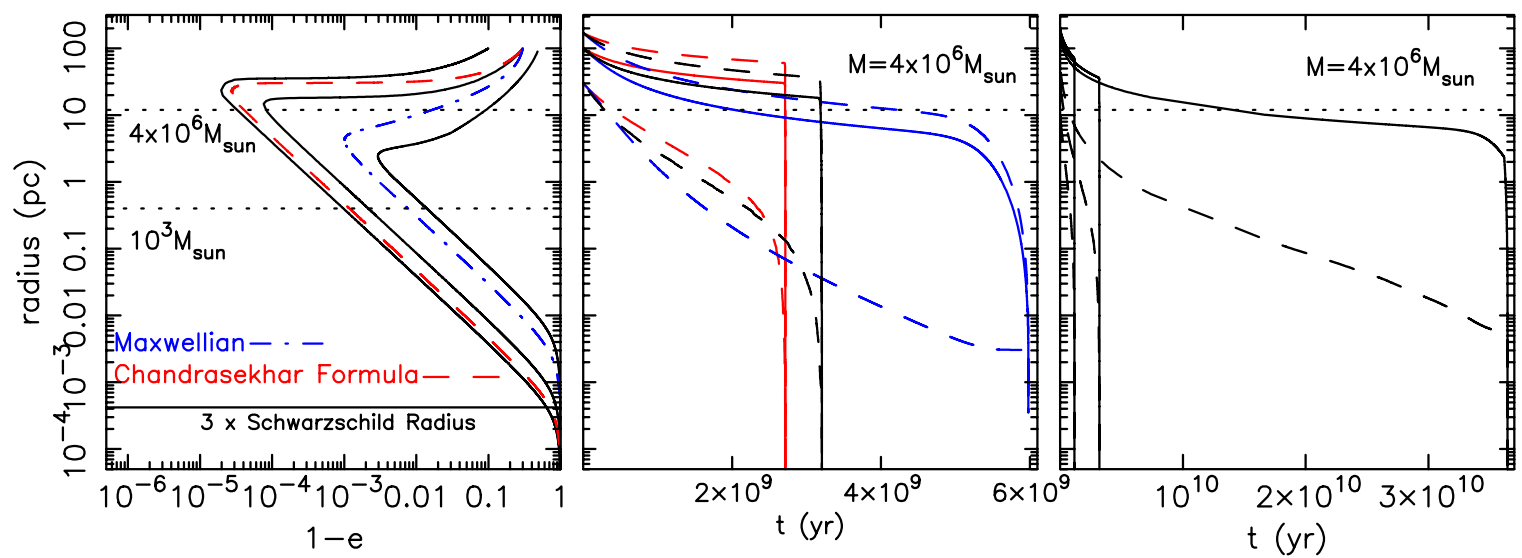

Figure 23. Left panel: orbital evolution in the $a,(1-e)$ plane for a massive object in the M87 core starting from various eccentricities $e_{\text {in }}=(0.5,0.7,0.9)$ and from an initial semimajor axis $a_{\mathrm{in}}=100 \mathrm{pc}$. Dynamical friction and gravitational wave energy losses are both included. Dotted lines represent the radii at which the stellar mass enclosed in the orbit is $10^{3} M_{\odot}$ (lower curve) or $4 \times 10^{6} M_{\odot}$ (upper curve). Red and blue lines are obtained, respectively, from the standard Chandrasekhar formula (18), which neglects fast-moving stars and from Equation (31) that assumes in addition a Maxwellian velocity distribution. Black curves are based on the more general Equation (40). Horizontal solid line gives the ISCO radius for a non-spinning hole (i.e., six gravitational radii). Central panel: time evolution of orbital semimajor axis (solid lines), apoapsis (upper-dashed curves), and periapsis (lower-dashed curves) in the three integrations with $e_{\text {in }}=0.7$ performed using: (a) the correct formula that includes the contribution from fast-moving stars (black curves), (b) Equation (18) in which only stars moving slower than the test mass contribute to the frictional drag (red curves), (c) Equation (31) which assumes a Maxwellian distribution of velocities (blue curves). Right panel: time evolution of apoapsis (solid lines) and orbital periapsis (dashed lines) for a $4 \times 10^{6} M_{\odot}$ black hole.

infall in this case result in a longer lifetime of the massive binary (central panel). The red curves in Figure 23 were obtained using Equation (18), which allows for a non-Maxwellian velocity distribution but neglects the contribution to the frictional drag from stars moving faster than the sinking black hole. This approximation also results in a very different evolution when compared to the more correct treatment (black curve). Due to the smaller frictional drag, the standard treatment produces a slower decay of the orbital semimajor axis but a much faster evolution of the eccentricity, which in turns results in a shorter lifetime of the black hole binary. The right panel of Figure 23 shows the time evolution of orbital radius when $M=4 \times 10^{6} M_{\odot}$. In a shallow cusp near a SMBH, dynamical friction is very inefficient; this results in a very long sinking time. Starting from $100 \mathrm{pc}$, black holes with masses $M \lesssim 4 \times 10^{6} M_{\odot}$ do not reach the center of the galaxy in a Hubble time unless their orbit has a substantial initial eccentricity $\left(e_{\text {in }} \gtrsim 0.7\right)$. We note however that such large eccentricities could be difficult to retain at these radii due to orbital circularization that occurs outside the sphere of influence of the central SMBH.

Cosmological simulations predict that a giant elliptical like M87 accreted about four Milky Way sized galaxies over the last $\sim 5$ Gyr (Fakhouri et al. 2010). The long sinking timescale found in Figure 23 suggest therefore that, at the present epoch, brightest cluster galaxies may still contain a few massive black holes or even satellite galaxies (see below) moving through their extended cores. Although non-active secondary black holes could be very difficult to detect directly, such systems would be a possible source of jet precession in the active galactic nucleus of the central galaxy (Romero et al. 2000) or they could induce a detectable displacement between the galactic photo-center and its nuclear point source (Batcheldor et al. 2010).

In the computations presented above the infalling object was treated as a test particle of fixed mass. However, in a massive galaxy like M87 the central density is low enough that the infalling black hole may retain a significant fraction of stars from its host galaxy (because tidal forces are small). If stalling occurs, then one or more satellites may remain in the core of the central galaxy for a time significantly longer than a Hubble time.
To address this possibility, we integrated the equations of motion of a satellite galaxy in a fixed potential including the contribution of dynamical friction and the effect of tidal truncation (e.g., Antonini et al. 2011). The tidally truncated mass of the satellite galaxy $\left(m_{T}\right)$ is related to its limiting radius $\left(r_{T}\right)$ via

$$
G m_{T} \approx \frac{1}{2} \sigma^{2} r_{T},
$$

with $\sigma$ the one dimensional central velocity dispersion. The mass of the satellite SMBH is fixed by $\sigma$ through the $M-\sigma$ relation (Gültiken et al. 2009):

$$
M=1.3 \times 10^{8}\left(\sigma / 200 \mathrm{~km} \mathrm{~s}^{-1}\right)^{4.24} .
$$

The tidal radius can then be related to the potential $\phi$ and density $\rho$ of the central galaxy by (e.g., King 1962)

$$
r_{T}=\frac{1}{\sqrt{2}} \sigma\left[\frac{3}{r}\left(\frac{d \phi}{d r}\right)-4 \pi G \rho\right]^{-1 / 2} .
$$

Using for the central galaxy the mass distribution of Equation (35), we find

$$
\frac{d \phi}{d r}=\frac{8 \pi}{5} G \rho_{0} r_{0}\left(\frac{r}{r_{0}}\right)^{\frac{1}{2}}+\frac{G M_{\bullet}}{r^{2}},
$$

where $\rho_{0}=35 M_{\odot} \mathrm{pc}^{-3}$ and $r_{0}=600 \mathrm{pc}$. This gives a limiting radius

$$
r_{T}=\frac{1}{\sqrt{2}} \sigma\left[\frac{4 \pi}{5} G \rho_{0}\left(\frac{r}{r_{0}}\right)^{-1 / 2}+\frac{3 G M_{\bullet}}{r^{3}}\right]^{-1 / 2}
$$

and a tidally truncated mass from Equation (49). Adopting an initial distance of $600 \mathrm{pc}$ and $\sigma=94 \mathrm{~km} \mathrm{~s}^{-1}$ (corresponding to $M=4 \times 10^{6} M_{\odot}$ ) we find $m_{T}=4.6 \times 10^{7} M_{\odot}$ and $r_{T}=45 \mathrm{pc}$.

Figure 24 plots the orbital evolution of satellites with initial orbital radius $r=600 \mathrm{pc}$ and different values of the central velocity dispersion $\sigma=(50,94,200) \mathrm{km} \mathrm{s}^{-1}$ corresponding to $M=\left(3 \times 10^{5}, 4 \times 10^{6}, 10^{8}\right) M_{\odot}$. In the core of a giant elliptical 


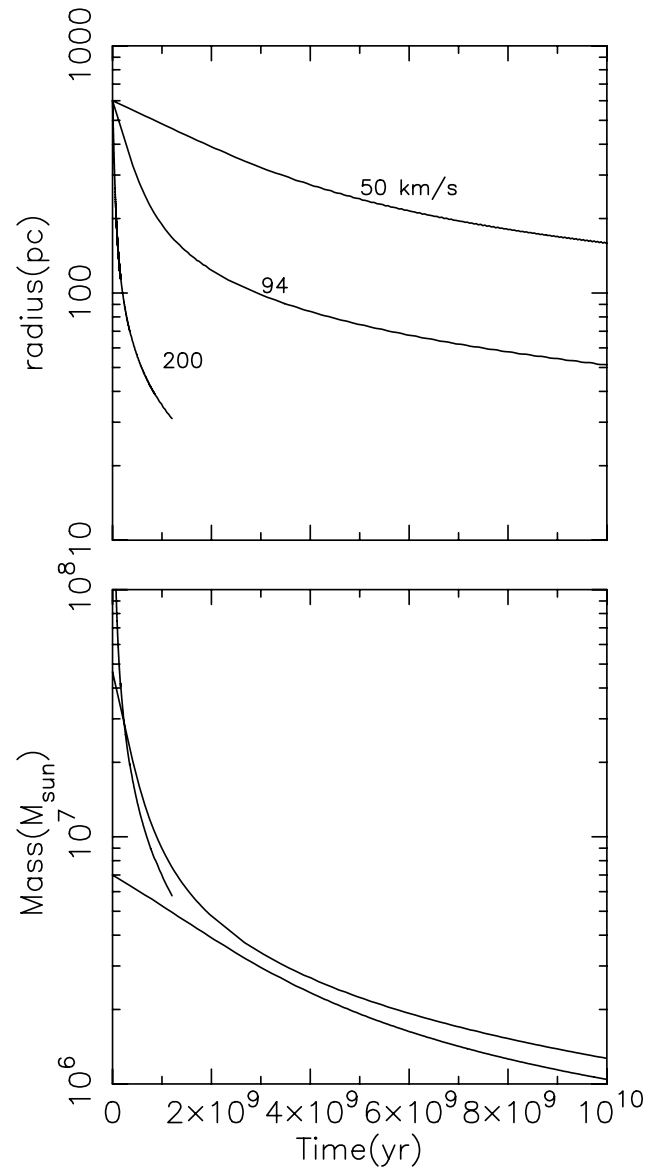

Figure 24. Upper panel displays the orbital decay of satellite galaxies with different central velocity dispersions (or central black hole masses) into the core of M87. The evolution of the mass in stars of the infalling galaxies, as determined by the central galaxy tidal field, is given in the lower panel.

galaxy like M87, the time to reach the center for galaxies with $\sigma \lesssim 100 \mathrm{~km} \mathrm{~s}^{-1}$ is evidently longer than a Hubble time.

First-ranked galaxies in clusters are often observed to contain multiple "nuclei," which may be identified with the tidally truncated remains of inspiralling galaxies (Merritt 1984).

\section{CONCLUSIONS}

In this paper, we considered the orbital evolution of massive objects in nuclei with shallow density profiles around SMBHs. Our principle results are summarized below.

1. Orbital evolution can be very sensitive to the details of the stellar distribution. In models with a flat central density profile, $\rho \sim r^{-\gamma}, \gamma \approx 0.5$, the dynamical-friction timescale is much longer than in models with a steep cusp due to the lack of low-velocity stars. The standard formula predicts that the inspiraling body will stall at a radius that is roughly $1 / 2$ the core radius.

2. Orbital eccentricity increases rapidly when the periapsis falls inside the core. If the inspiralling body is initially at $r_{\text {bh }}$ with $e_{\text {in }} \gtrsim 0.5$, its orbital eccentricity can become very large $(\gtrsim 0.9)$ by the time the orbit lies entirely inside the core.

3. Using $N$-body simulations, we found that the frictional force never falls precisely to zero. When the contribution of the fast-moving stars is included in the expression for the dynamical-friction force, and (if appropriate) the changes induced by the massive body on the stellar distribution are taken into account, Chandrasekhar's theory reproduces the decay observed in the $N$-body simulations very accurately. On the other hand, a straightforward application of Chandrasekhar's formula in its standard form can give misleading results.

4. If the mass of the inspiralling object is sufficiently large, it promotes the diffusion of stars into the phase-space region that was initially nearly empty, increasing the dynamicalfriction force. A low-density core is again regenerated as the object displaces these stars.

5. We derived an estimate of the Coulomb logarithm without any particular assumptions about the velocity distribution of field stars (e.g., that it follows a Maxwellian distribution), and in the region outside the core, where the standard dynamical-friction formula (18) accurately represents the motion. We obtained $\ln \Lambda \approx 6.5$, consistent with previous work.

6. We studied the location and evolution of the gravitational wake that the inspiralling body induces in the stellar background. Outside the core, the peak in the overdensity lies close to the massive body at $-20<\theta \lesssim 0^{\circ}$, independent of $M$, and the amplitude of the overdensity increases with black hole mass. After the massive body enters the core, the density maximum decreases. This is consistent with the fact that the frictional drag is greatly reduced inside the shallow cusp.

7. In the absence of a steep central density cusp, the time for stellar-mass BHs to reach the center of the Milky Way from a starting radius of order $1 \mathrm{pc}$ can easily exceed $10 \mathrm{Gyr}$. We computed the evolution of a population of stellar BHs as they segregate to the $\mathrm{GC}$, including the frictional force from the fast-moving stars. We found that, in models with parsec-scale cores, even after $10 \mathrm{Gyr}$, the density of black holes can remain substantially lower than that in stars at all radii. We conclude that it would be unjustified to assume that the massive remnants have yet reached a steady-state distribution at the GC.

8. Secondary black holes reach the gravitational-radiationdominated regime on orbits that are typically very eccentric. However, we found that even initially moderate eccentricities would result in non-negligible eccentricities at the moment the binary black hole enters the sensitivity window of planned space-based interferometers. This in turn would require non-circular templates for $\mathrm{GW}$ data analysis.

As a final remark, we recommend using Equation (36) for the study of the inspiral of massive objects in GCs.

This work was supported by the National Science Foundation under grant nos. AST 08-07910 and 08-21141 and by the National Aeronautics and Space Administration under grant no. NNX-07AH15G. We thank the referee $\mathrm{M}$. Weinberg for comments that helped to improve the paper, and we are indebted to T. Alexander, B. Kocsis, H. Perets, and E. Vasiliev for useful discussions.

\section{REFERENCES}

Alexander, T. 2005, Phys. Rep., 419, 65

Alexander, T., \& Hopman, C. 2009, ApJ, 697, 1861

Amaro-Seoane, P., Gair, J. R., Freitag, M., et al. 2007, Class. Quantum Grav., 24, 113

Antonini, F., Capuzzo-Dolcetta, R., Mastrobuono-Battisti, A., \& Merritt, D. 2011, arXiv:1110.5937

Bahcall, J. N., \& Wolf, R. A. 1977, ApJ, 216, 883 
Barack, L., \& Cutler, C. J. 2004, Phys. Rev. D, 69, 082005

Bartko, H., Martins, F., Trippe, S., et al. 2010, ApJ, 708, 834

Batcheldor, D., Robinson, A., Axon, D. J., Perlman, E. S., \& Merritt, D. 2010, ApJ, 717, L6

Baumgardt, H., Gualandris, A., \& Portegies Zwart, S. 2006, MNRAS, 372, 174

Binney, J. J., \& Evans, N. W. 2001, MNRAS, 327, L27

Borriello, A., \& Salucci, P. 2001, MNRAS, 323, 285

Buchholz, R. M., Schödel, R., \& Eckart, A. 2009, A\&A, 499, 483

Chandrasekhar, S. 1943, ApJ, 97, 255

Cora, S. A., Muzzio, J. C., \& Vergne, M. M. 1997, MNRAS, 289, 253

Danby, J. J. A., \& Camm, G. L. 1957, MNRAS, 117, 50

Do, T., Ghez, A. M., Morris, M. R., et al. 2009, ApJ, 703, 1323

Ebisuzaki, T., Makino, J., \& Okumura, S. K. 1991, Nature, 354, 212

Faber, S. M., Tremaine, S., Ajhar, E. A., et al. 1997, AJ, 114, 1771

Fakhouri, O., Ma, C., \& Boylan-Kolchin, M. 2010, MNRAS, 406, 2267

Fehlberg, E. 1968, NASA Technical Report, TR T-287

Ferrarese, L., van den Bosch, F. C., Ford, H. C., Jaffe, W., \& O'Connell, R. W. 1994, AJ, 108, 1598

Freitag, M., Amaro-Seoane, P., \& Kalogera, V. 2006, ApJ, 649, 91

Fukushige, T., Ebisuzaki, T., \& Makino, J. 1992, PASJ, 44, 281

Gaburov, E., Harfst, S., \& Portegies Zwart, S. 2009, New Astron., 14, 630

Gebhardt, K., Richstone, D., Ajhar, E. A., et al. 1996, AJ, 112, 105

Ghez, A. M., Salim, S., Weinberg, N. N., et al. 2008, ApJ, 689, 1044

Gillessen, S., Eisenhauer, F., Trippe, S., et al. 2009, ApJ, 692, 1075

Goerdt, T., Moore, B., Read, J. I., Stadel, J., \& Zemp, M. 2006, MNRAS, 368, 1073

Gould, A., \& Quillen, A. C. 2003, ApJ, 592, 935

Gualandris, A., \& Merritt, D. 2008, ApJ, 678, 780

Gualandris, A., \& Merritt, D. 2011, arXiv:1107.4095

Gültiken, D. O., Richstone, D. O., Gebhardt, K., et al. 2009, ApJ, 698, 198

Harfst, S., Gualandris, A., Merritt, D., et al. 2007, New Astron., 12, 357

Hernandez, X., \& Gilmore, G. 1998, MNRAS, 297, 517

Hopman, C., \& Alexander, T. 2006, ApJ, 645, L133

Hernquist, L., \& Weinberg, M. D. 1989, MNRAS, 238, 407

Hughes, S. A. 2003, Ann. Phys., 303, 142

Ibata, R. A., \& Lewis, G. F. 1998, ApJ, 500, 575

Inoue, S. 2009, MNRAS, 397, 709

Just, A., Khan, F. M., Berczik, P., Ernst, A., \& Spurzem, R. 2011, MNRAS, 411,653

Kalnajs, A. J. 1972, in IAU Coll. 10, Gravitational N-body Problems, ed. M. Lecar (Dordrecht: Reidel), 13

King, I. R. 1962, AJ, 67, 471

Lauer, T. R., Ajhar, E. A., Byun, Y.-I., et al. 1995, AJ, 110, 2622

Lauer, T. R., Faber, S. M., Lynds, R. C., et al. 1992, AJ, 103, 703

Lin, D. N. C., \& Tremaine, S. 1983, ApJ, 264, L364

Löckmann, U., \& Baumgardt, H. 2008, MNRAS, 384, 323
Lynden-Bell, D., \& Kalnajs, A. J. 1972, MNRAS, 157, 1

Makino, J., \& Funato, Y. 2004, ApJ, 602, 93

Marochnik, L. S. 1968, SvA, 11, 873

Merritt, D. 1984, ApJ, 280, L5

Merritt, D. 2001, ApJ, 556, 245

Merritt, D. 2006, ApJ, 648, 976

Merritt, D. 2010, ApJ, 718, 739

Merritt, D. 2012, Black Holes and the Dynamics of Galactic Nuclei (Princeton, NJ: Princeton Univ. Press)

Merritt, D., Alexander, T., Mikkola, S., \& Will, C. M. 2011, Phys. Rev. D, 84 044024

Merritt, D., \& Szell, A. 2006, ApJ, 648, 890

Milosavljević, M., \& Merritt, D. 2001, ApJ, 563, 34

Morris, M. 1993, ApJ, 408, 496

Mulder, W. A. 1983, A\&A, 117, 9

Murai, T., \& Fujimoto, M. 1980, PASJ, 32, 581

Oh, S., Kim, S. S., \& Figer, D. F. 2009, J. Korean Astron. Soc., 42, 17

Palmer, P. L., \& Papaloizou, J. 1985, MNRAS, 215, 691

Peters, P. C. 1964, Phys. Rev., 136, 1224

Peters, P. C., \& Mathews, J. 1963, PhRv, 131, 435

Porter, E., \& Sesana, A. 2010, arXiv:1005.5296

Quinlan, G. D. 1996, New Astron., 1, 35

Quinlan, G. D., \& Hernquist, L. 1997, New Astron., 2, 533

Rauch, K. P., \& Tremaine, S. 1996, New Astron., 1, 149

Read, J. I., Goerdt, T., Moore, B., et al. 2006, MNRAS, 373, 1451

Romero, G. E., Chajet, L., Abraham, Z., \& Fan, J. H. 2000, A\&A, 360, 57

Rosenbluth, M. N., MacDonald, W. M., \& Judd, D. L. 1957, Phys. Rev., 107, 1

Spekkens, K., Giovanelli, R., \& Haynes, M. P. 2005, AJ, 129, 2119

Spinnato, P. F., Fellhauer, M., \& Portegies Zwart, S. F. 2003, MNRAS, 344, 22

Spitzer, L. 1987, Dynamical Evolution of Globular Clusters (Princeton, NJ: Princeton Univ. Press), 191

Szell, A., Merritt, D., \& Kevrekidis, I. G. 2005, Phys. Rev. Lett., 95, 081102

Tremaine, S., \& Weinberg, M. D. 1984, MNRAS, 209, 729

van den Bosch, F. C., Lewis, G. F., Lake, G., \& Stadel, J. 1999, ApJ, 515, 50

Vesperini, E., \& Weinberg, M. D. 2000, ApJ, 534, 598

Weinberg, M. D. 1985, MNRAS, 213, 451

Weinberg, M. D. 1986, ApJ, 300, 93

Weinberg, M. D. 1989, MNRAS, 239, 549

Weinberg, M. D. 2004, arXiv:astro-ph/0404169

Weinberg, M. D., \& Katz, N. 2007, MNRAS, 375, 425

Weinberg, M. D., \& Katz, N. 2002, ApJ, 580, 627

White, M. L. 1949, ApJ, 109, 159

White, S. D. M. 1983, ApJ, 274, 53

Woosley, S. E., Heger, A., \& Weaver, T. A. 2002, Rev. Mod. Phys., 74, 1015

Young, P. J., Westphal, J. A., Kristian, J., Wilson, C. P., \& Landauer, F. P. 1978, ApJ, 221, 721 